NASA/TM-2013-216546

Note

Glove-Enabled Computer Operations (GECO): Design and Testing of an Extravehicular Activity Glove Adapted for Human-Computer Interface

Richard J. Adams, Aaron Olowin, and Eileen Krepkovich

Barron Associates Incorporated, Charlottesville, Virginia

Blake Hannaford and Jack I. C. Lindsay

University of Washington, Seattle, Washington

Peter Homer

Flagsuit LLC, Southwest Harbor, Maine

James T. Patrie

Statistical Consultant, Charlottesville, Virginia

O. Scott Sands

Glenn Research Center, Cleveland, Ohio 


\section{NASA STI Program . . . in Profile}

Since its founding, NASA has been dedicated to the advancement of aeronautics and space science. The NASA Scientific and Technical Information (STI) program plays a key part in helping NASA maintain this important role.

The NASA STI Program operates under the auspices of the Agency Chief Information Officer. It collects, organizes, provides for archiving, and disseminates NASA's STI. The NASA STI program provides access to the NASA Aeronautics and Space Database and its public interface, the NASA Technical Reports Server, thus providing one of the largest collections of aeronautical and space science STI in the world. Results are published in both non-NASA channels and by NASA in the NASA STI Report Series, which includes the following report types:

- TECHNICAL PUBLICATION. Reports of completed research or a major significant phase of research that present the results of NASA programs and include extensive data or theoretical analysis. Includes compilations of significant scientific and technical data and information deemed to be of continuing reference value. NASA counterpart of peer-reviewed formal professional papers but has less stringent limitations on manuscript length and extent of graphic presentations.

- TECHNICAL MEMORANDUM. Scientific and technical findings that are preliminary or of specialized interest, e.g., quick release reports, working papers, and bibliographies that contain minimal annotation. Does not contain extensive analysis.

- CONTRACTOR REPORT. Scientific and technical findings by NASA-sponsored contractors and grantees.
- CONFERENCE PUBLICATION. Collected papers from scientific and technical conferences, symposia, seminars, or other meetings sponsored or cosponsored by NASA.

- SPECIAL PUBLICATION. Scientific, technical, or historical information from NASA programs, projects, and missions, often concerned with subjects having substantial public interest.

- TECHNICAL TRANSLATION. Englishlanguage translations of foreign scientific and technical material pertinent to NASA's mission.

Specialized services also include creating custom thesauri, building customized databases, organizing and publishing research results.

For more information about the NASA STI program, see the following:

- Access the NASA STI program home page at http://www.sti.nasa.gov

- E-mail your question to help@sti.nasa.gov

- Fax your question to the NASA STI Information Desk at 443-757-5803

- Phone the NASA STI Information Desk at 443-757-5802

- Write to: STI Information Desk NASA Center for AeroSpace Information 7115 Standard Drive Hanover, MD 21076-1320 
NASA/TM-2013-216546

Glove-Enabled Computer Operations (GECO): Design and Testing of an Extravehicular Activity Glove Adapted for Human-Computer Interface

Richard J. Adams, Aaron Olowin, and Eileen Krepkovich

Barron Associates Incorporated, Charlottesville, Virginia

Blake Hannaford and Jack I. C. Lindsay

University of Washington, Seattle, Washington

Peter Homer

Flagsuit LLC, Southwest Harbor, Maine

James T. Patrie

Statistical Consultant, Charlottesville, Virginia

O. Scott Sands

Glenn Research Center, Cleveland, Ohio

Prepared for the

43rd International Conference on Environmental Systems

sponsored by the American Institute for Aeronautics and Astronautics

Vail, Colorado, July 14-18, 2013

National Aeronautics and

Space Administration

Glenn Research Center

Cleveland, Ohio 44135 


\section{Acknowledgments}

We gratefully acknowledge the support of NASA Glenn Research Center. Work on the Glove-Enabled Computer Operations (GECO) System was performed under contract NNX11CA55C. Significant contributors to the GECO software development included John Reilly and Rebecca Bird (Barron Associates, Incorporated).

Trade names and trademarks are used in this report for identification only. Their usage does not constitute an official endorsement, either expressed or implied, by the National Aeronautics and Space Administration.

Level of Review: This material has been technically reviewed by technical management.

Available from

NASA Center for Aerospace Information 7115 Standard Drive

Hanover, MD 21076-1320
National Technical Information Service 5301 Shawnee Road Alexandria, VA 22312

Available electronically at http://www.sti.nasa.gov 


\title{
Glove-Enabled Computer Operations (GECO): Design and Testing of an Extravehicular Activity Glove Adapted for Human-Computer Interface
}

\author{
Richard J. Adams, Aaron Olowin, and Eileen Krepkovich \\ Barron Associates Incorporated \\ Charlottesville, Virginia 22901 \\ Blake Hannaford and Jack I. C. Lindsay \\ University of Washington \\ Seattle, Washington 98195 \\ Peter Homer \\ Flagsuit LLC \\ Southwest Harbor, Maine 04679 \\ James T. Patrie \\ Statistical Consultant \\ Charlottesville, Virginia 22903 \\ O. Scott Sands \\ National Aeronautics and Space Administration \\ Glenn Research Center \\ Cleveland, Ohio 44135
}

\begin{abstract}
The Glove-Enabled Computer Operations (GECO) system enables an extravehicular activity (EVA) glove to be dual-purposed as a human-computer interface device. This paper describes the design and human participant testing of a right-handed GECO glove in a pressurized glove box. As part of an investigation into the usability of the GECO system for EVA data entry, twenty participants were asked to complete activities including (1) a Simon Says Games in which they attempted to duplicate random sequences of targeted finger strikes and (2) a Text Entry activity in which they used the GECO glove to enter target phrases in two different virtual keyboard modes. In a within-subjects design, both activities were performed both with and without vibrotactile feedback. Participants' mean accuracies in correctly generating finger strikes with the pressurized glove were surprisingly high, both with and without the benefit of tactile feedback. Five of the subjects achieved mean accuracies exceeding $99 \%$ in both conditions. In Text Entry, tactile feedback provided a statistically significant performance benefit, quantified by characters entered per minute, as well as reduction in error rate. Secondary analyses of responses to a NASA Task Loader Index (TLX) subjective workload assessments reveal a benefit for tactile feedback in GECO glove use for data entry. This first-ever investigation of employment of a pressurized EVA glove for human-computer interface opens up a wide range of future applications, including text "chat" communications, manipulation of procedures/checklists, cataloguing/annotating images, scientific note taking, human-robot interaction, and control of suit and/or other EVA systems.
\end{abstract}

\subsection{Introduction}

Future space suits may be equipped with on-board computing, networking, and helmet-mounted graphical displays (Refs. 1 and 2) to provide astronauts with access to some of the same "apps" that terrestrial smart phone users now take for granted. These may include textual and voice communications, 
map-based navigation, video/image acquisition, document viewing/editing, and news/weather alerts. Currently, few practical solutions exist to provide the point-and-click and rapid textual/numeric data entry capabilities that many of these applications require. The GECO system permits gloved hands to be used as human-computer interface devices by crewmembers during suited EVA, emulating the functionality normally provided by a standard mouse, keyboard, or other conventional input method. It is the embodiment of the tactile data entry capability identified in NASA's integrated technology roadmap (Ref. 3).

Traditional interfaces such as a keypad/keyboard, mouse, and touch screen displays are poorly adapted for use by a hand confined with a space suit glove. EVA gloves, dating from the Gemini program to those used today on the International Space Station, have been constructed of at least three layers. Closest to the hand is an impermeable bladder that maintains the pressurized environment inside of the suit. The pressure-induced loads are carried by a fabric restraint layer, augmented by metal reinforcements (such as a palm bar). Together, the bladder and restraint layer comprise the "pressure glove," designed to achieve near-constant volume, and thus minimize joint torques and fatigue, during use. Outside of the pressure glove, a thermal and micrometeoroid garment (TMG) provides protection from punctures/abrasion, temperature extremes, and penetration by micrometeoroid particles (Ref. 4).

Unfortunately, these elements which afford protection from environmental hazards impede the dexterity, tactility, and mobility required to manipulate traditional human-machine interfaces (Ref. 5).

The GECO system takes a different approach, leveraging EVA glove design features as platforms for instrumentation and feedback elements that turn the gloves themselves into human-computer interface devices. Rather than being barriers between the human operator and a data entry device, the GECO gloves ARE data entry devices. Each finger of a glove can be thought of as a button that can be mapped to any number of functions (e.g., a mouse click, a keyboard strike, or a control toggle). Hand translation can be interpreted as the movement of a mouse, driving cursor position on a graphical user interface (GUI), or a change in hand position on a virtual keyboard, altering the mapping between fingers and keys. The addition of programmable vibrotactile feedback enriches the interface by replicating the haptic sensations that humans intuitively associate with an input device (e.g., the resistance/recoil of a button, the feel of an edge). Potential EVA applications include text chat communications (enabling time delayed and low bandwidth dialog), working with procedures/checklists (e.g., scrolling, moving a focus bar, marking steps/instruction as complete), cataloguing/annotating images (e.g., during geological exploration, survey, and sample collection), note taking, human-robot interaction (e.g., shared autonomy, supervisory control, and/or direct tele-manipulation), or simply replacing Apollo-era suit controls.

The introduction of tactile feedback within the GECO design aims to increase user performance and reduce mental workload. User displays often over-rely on other perceptual channels (in particular visual), resulting in taxed cognitive resources in an EVA environment already saturated with information sources. The consequences of information overload can include poor task performance, poor multi-tasking efficiency, and higher incidence of operator errors (Ref. 6). Compared to visual and audio, touch is an underused sensory pathway. For EVA applications, this creates an opportunity for presentation of information with reduced cognitive loading.

Speech-to-text and voice recognition present potential alternatives to the manual/touch-based approach enabled by GECO, with the possibility of providing hands-free and heads-up operation. Previous research employing the MX-2 neutral buoyancy space suit analogue included speech recognition, combined with feedback through both auditory and graphical display, to enable both advanced controls and human-robotic interaction (Refs. 7 and 8). Spoken dialogue system have been tested by NASA to support human-computer interaction, including recent strides in addressing robustness to in-suit noise and discernment of context (Refs. 9 and 10). Voice/aural-based systems have the potential to be effective in well-structured tasks in which the application software has been tailored to the strengths and weaknesses of the interface. They are, however, mal-suited for activities that involve spatial information. Common GUI interactions as fundamental as clicking a button, moving a slider bar, selecting an icon, or positioning a cursor in a text field range from problematic to extremely difficult using voice. These limitations explain, in part, why after decades of development such systems have yet to challenge the dominance of the keyboard and mouse in the consumer market. Speech-to-text and voice 
recognition systems will almost certainly be part of future EVA systems, but will be highly limiting if they are the only data entry mode available. The most effective solution will likely be a synergistic combination of human-machine interaction technologies, including audio, manual/tactile, and visual modalities.

Humans' ability to process visual information at an extremely rapid rate make graphical display an essential component of an effective computer interface. Helmet displays are identified in NASA's integrated technology roadmap (Ref. 3) as part of the future suit architecture, and are the subject of significant past and current research (Ref. 2). Unfortunately, without an effective data entry method, the display is only half a solution. Taking advantage of a graphical environment for computer interface (e.g., Mac OS, Windows, and Ubuntu) requires the ability to point, select, and move objects. The modern computer mouse, in fact, emerged from this requirement. Its enduring popularity is a testimony to the primacy of the hand as the most effective medium through which spatial information and discrete events are communicated from a human to a computer. The emergence of the touchscreen as the primary input modality for smartphones only reinforces this premise. In the EVA environment, where neither the mouse nor the touch screen are practical, the gloves on a crewmember's hands can fill this role.

The GECO concept was initially explored in an investigation of data entry performance employing a soccer goalie glove (as a crude unpressurized EVA glove surrogate) with sensory and tactile feedback components (Ref. 11). In a text entry task performed by twenty participants, users entered characters significantly faster with tactile feedback than without. Both objective (character entry and error rates) and subjective (Likert-scale questionnaire) measures showed a statistically-significant relationship between tactile feedback and performance. The success of this initial prototyping and experimental activity led to development of a more flight-like space suit glove and experimentation under pressurized conditions, described herein.

\subsection{Design}

The GECO system employs a bladder-restraint pressure glove assembly based on a design by Flagsuit LLC (Southwest Harbor, ME) that was awarded first prize in two NASA Astronaut Glove Centennial Challenges (in 2007 and 2009). Finger mobility is provided via a patented fabric joint, providing lowtorque flexion/extension. Tactile actuators (C-10 Linear Resonant Actuator, from Precision Microdrives Inc.), custom integration features (Ref. 12), and wiring harnesses are all mounted on the outside of the restraint layer (dorsal side) above each finger to enable rendering of vibratory cues. The design, shown in Figure 1, takes advantage of the stiffness of the pressurized glove to carry vibrations from these actuators to the user's finger tips, thus permitting placement of the components in a manner that does not interfere with the glove's normal use for manual manipulation. Typical vibrotactile feedback for a "button click" event takes the form of two $175 \mathrm{~Hz}$ square wave pulses of $25 \mathrm{~ms}$ duration and a $25 \mathrm{~ms}$ inter-pulse delay.

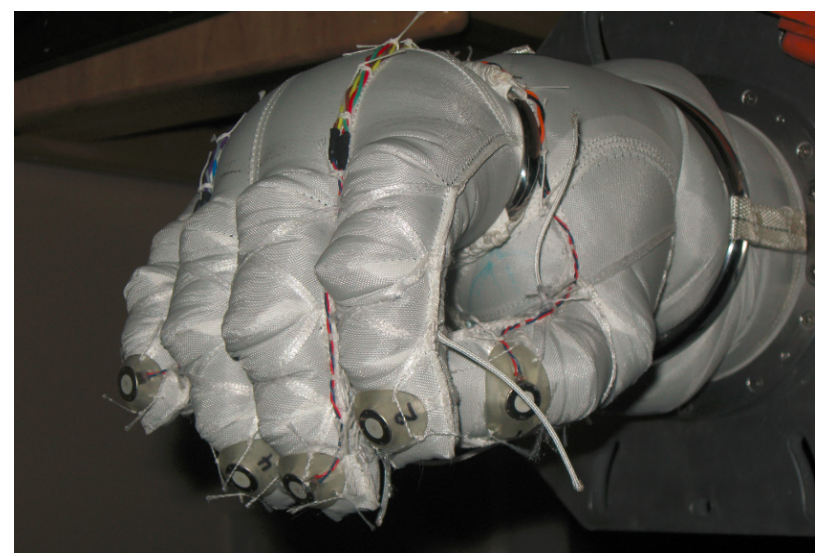

Figure 1.-GECO pressure glove with vibrotactile actuators. 

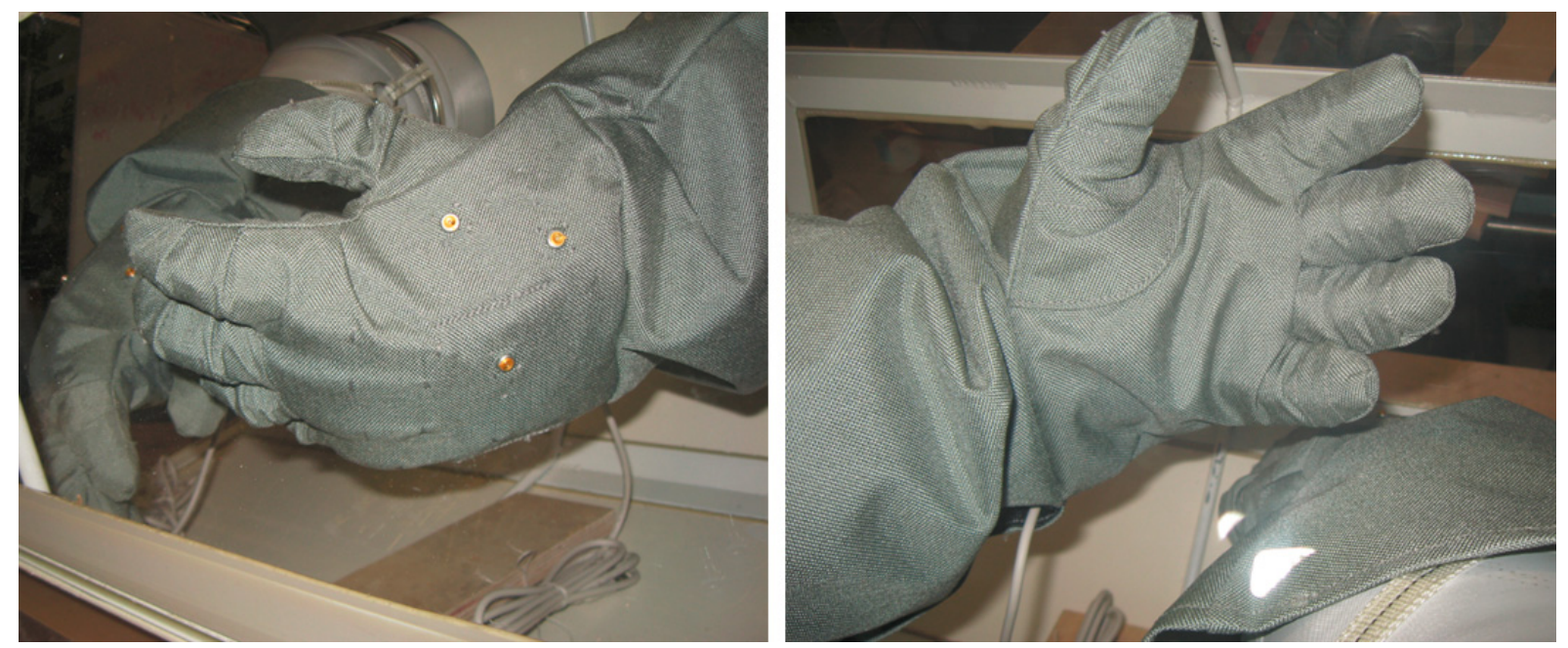

Figure 2.-Fully-integrated GECO glove.

All other GECO components are integrated into the surrogate TMG outer garment. These include a small custom printed circuit board (PCB) encompassing a microprocessor, signal conditioning, tactor driver, and power electronics. The microprocessor (STM32F103V8 from STMicroelectronics, Geneva, Switzerland) includes a central processing unit, random access memory, non-volatile memory, digital input/output, built-in timers, integrated analog-to-digital (A/D) conversion, serial communications, interintegrated circuit communications, and a Joint Test Action Group port for programming/debugging. During normal operations, all external data and power connections are via a single Universal Serial Bus (USB) cable to a personal computer. The fully-integrated glove is shown in Figure 2. The PCB, instrumentation, and harnessing are all integrated such that they do not interfere with, and are in fact are not even noticeable during, manual glove usage.

Finger flexion sensing is provided by $4.5 \mathrm{in}$. bend sensors (resistive ink patterned on a polyimide film) held within the surrogate TMG layer, aligned with each finger, using flexible tubing. Each sensor is connected to an operational amplifier voltage divider/filter circuit on the PCB through the GECO harness, providing the microprocessor, via its A/D channels, with a signal proportional to finger flexion angle. The microprocessor recognizes finger tapping motion by: (1) estimating individual finger speed as the change in finger position over a predetermined clock interval, (2) detecting speeds that exceed a predetermined positive speed threshold (rising edge), and (3) subsequently detecting a speed less than a predetermined negative speed threshold (falling edge), corresponding to an arresting motion. During the interval between detection of the rising and falling edge of an individual finger, the gesture recognition process is suspended for all other fingers on the associated hand. This temporary suspension prevents natural finger motion synergies (e.g., between the ring and little fingers) from generating erroneous events. The results of this algorithmic approach, applied to the instrumented GECO gloves pressurized at 4.3 pound per square inch differential (psid), are highlighted in Figure 3. The range of finger positions represent short tapping movements with each digit, progressing from thumb to little finger. Recognized finger "clicks" are shown as colored vertical lines; while finger position (bend sensor flexing) is shown as a series of asterisks representing normalized ADC counts. These data suggests a high rate of accuracy using this method, a hypothesis that was validated in the human subject testing.

Light emitting diodes (LEDs) on the back of the surrogate TMG enable tracking of hand motion by a suit-mounted camera, with a control circuit on the PCB providing on/off and/or continuous dimming. As a surrogate for suit-integrated optics, we employed an infrared camera (OptiTrack V120:SLIM from NaturalPoint, Inc.) which provides MPEG-compressed 640×480 pixel grayscale frames at $120 \mathrm{~Hz}$ through USB 2.0. Custom tracking software calculates hand position from the centroid locations of each glove's LEDs in the image frames. 


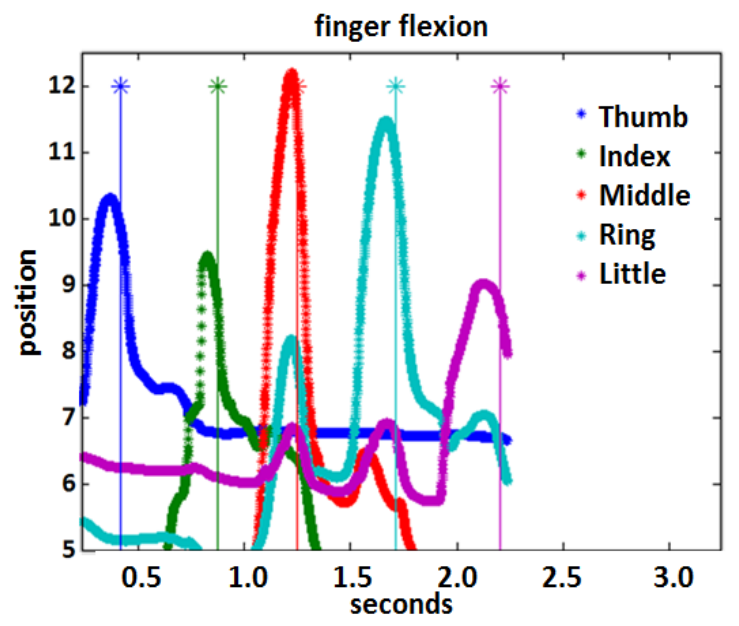

Figure 3.-GECO finger sensor data (asterisks indicate a registered button event).

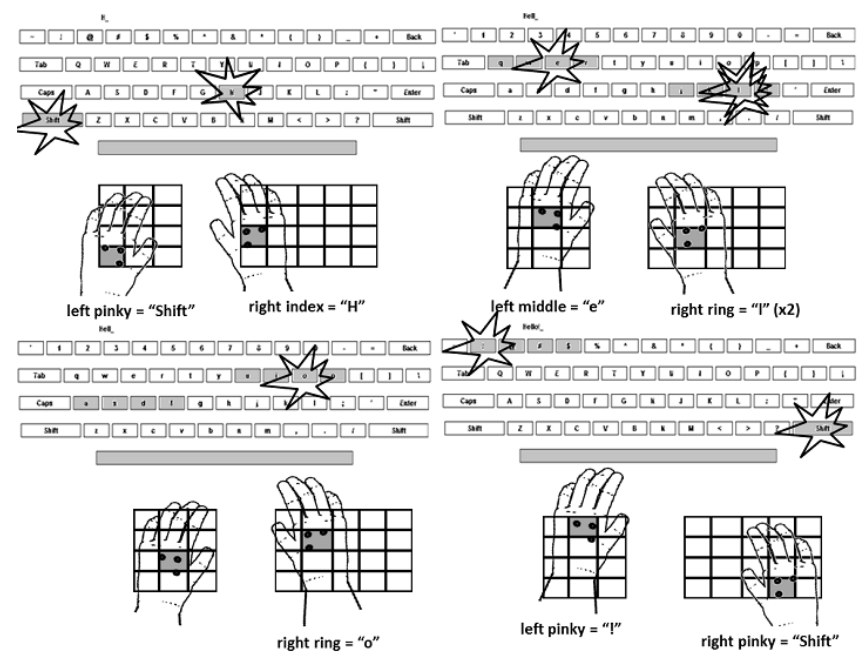

Figure 4.-GECO system in KEYBOARD mode.

A platform-agnostic Java-based GECO application programming interface (API) provides access to glove-generated events, sensor data, tactile event generation, and system control settings. The API has been used to implement automated test software (described below) and two different data entry modes: MOUSE and KEYBOARD. In MOUSE mode, the gloves emulate the functionality of a standard computer mouse, generating operating system events that are indistinguishable from the desktop analogue. Motion tracking permits hand position to drive the movement of a cursor on a visual display, while finger tapping motions are recognized and translated into mouse click events. Tactile feedback provides the physical sensation of button clicks, the edges of windows, and other GUI interactions.

In KEYBOARD mode, GECO emulates the functionality of a standard QWERTY keyboard. Hand motion controls the mapping from the fingers on right and left hands to keys on a virtual keyboard. When KEYBOARD mode is initialized, the fingers on the left hand map (from little to index finger) to "a," "s," "d," "f" and the fingers on the right hand to " $\mathrm{j}$, , "k," "l," ",,". The thumb on both hands is always mapped to the space bar. Translation of hand position changes the mapping to mimic the change of finger position on a physical keyboard. A forward motion with the left hand would thus change the mapping so that the index finger rests on the " $r$ " key. Finger tapping motions with any finger generates a keyboard stroke of the appropriate key. In this mode, tactile feedback to individual fingers provides the physical sensation of button clicks while a lighter, single-pulse "bump" effect indicates transition between finger-to-keyboard positions. Figure 4 illustrates use of the GECO system to generate the text "Hello!" in KEYBARD mode. 


\subsection{Human Participant Testing}

Human participant testing was conducted at Barron Associates, Inc. in Charlottesville, Virginia between August and October 2012 to evaluate the effectiveness of the GECO gloves as a human-computer interface. The research plan was approved and supervised by the NASA Institutional Review Board (IRB).

\subsection{Participants}

Twenty volunteers participated in the experiment. Volunteers were over the age of 21 and without physical impairment that would prevent normal use of the employed arm, hand, or fingers. To ensure adequate fit for the single prototype GECO glove, participants were screened to have palm width between 82 and $106 \mathrm{~mm}$ and hand length (from base of palm heel to tip of middle finger) between 197 and $226 \mathrm{~mm}$.

\subsection{Hypotheses}

Hypotheses to be investigated included:

Hypothesis 1-Participants make fewer errors with than without tactile feedback when using their gloved hand to perform simulated button clicks.

This hypothesis is tested through the Simon Says Game described below. The ability to accurately generate events with all five fingers of the GECO glove is essential for use as a data entry device. If users are able to effectively employ their gloved fingers to activate virtual buttons, any number of humancomputer interface modalities may be enabled. These range from simple switches or buttons, to more complex, and powerful, chorded keyboards.

Hypothesis 2-Participants are able to enter more characters with a lower error rate using their gloved hand with tactile feedback than without.

This hypothesis is tested in both the Hunt-and-peck and Touch-typing data entry modalities described below. Text entry is representative of a wide range of potential EVA applications, from text communications to geological sample cataloging. As the text entry activity requires translational hand movement and coordinated finger motion, it is also representative of other GUI interactions, such as icon clicking or widget controls.

Additionally, a range of secondary effects, such as inter-subject variability, training effects, and hand synergies were investigated through the activities.

\subsection{Materials and Software}

To approximate the impact of suit pressurization on manual dexterity, a test system was fabricated to permit operation at the same pressure differential (between the pressure volume inside and outside the bladder) experienced in the current NASA space suit "Phase VI" gloves employed on the International Space Station (Ref. 4). The setup includes a custom glove box (produced by Flagsuit LLC) consisting of a pressure-sealed container (hexagonal cylinder, $30 \mathrm{in}$. face-to-face, $32 \mathrm{in.} \mathrm{long)} \mathrm{made} \mathrm{of} \mathrm{steel} \mathrm{and} \mathrm{acrylic}$ sheet material; four access ports, into which space suit upper arm and glove assemblies can be inserted (Figure 5); and a diaphragm vacuum pump. Under normal operations, the inside of the glove box is evacuated to 4.3 psi below ambient atmospheric pressure, while the inside of the glove remains open to room ambient conditions, creating a 4.3 psid across the glove. When the glove is donned by a human subject, the human hand remains at room-level ambient pressure, but the glove feels and operates as it would on pressurized suit in the vacuum of space. A single right-handed GECO prototype glove was used for all evaluations. 


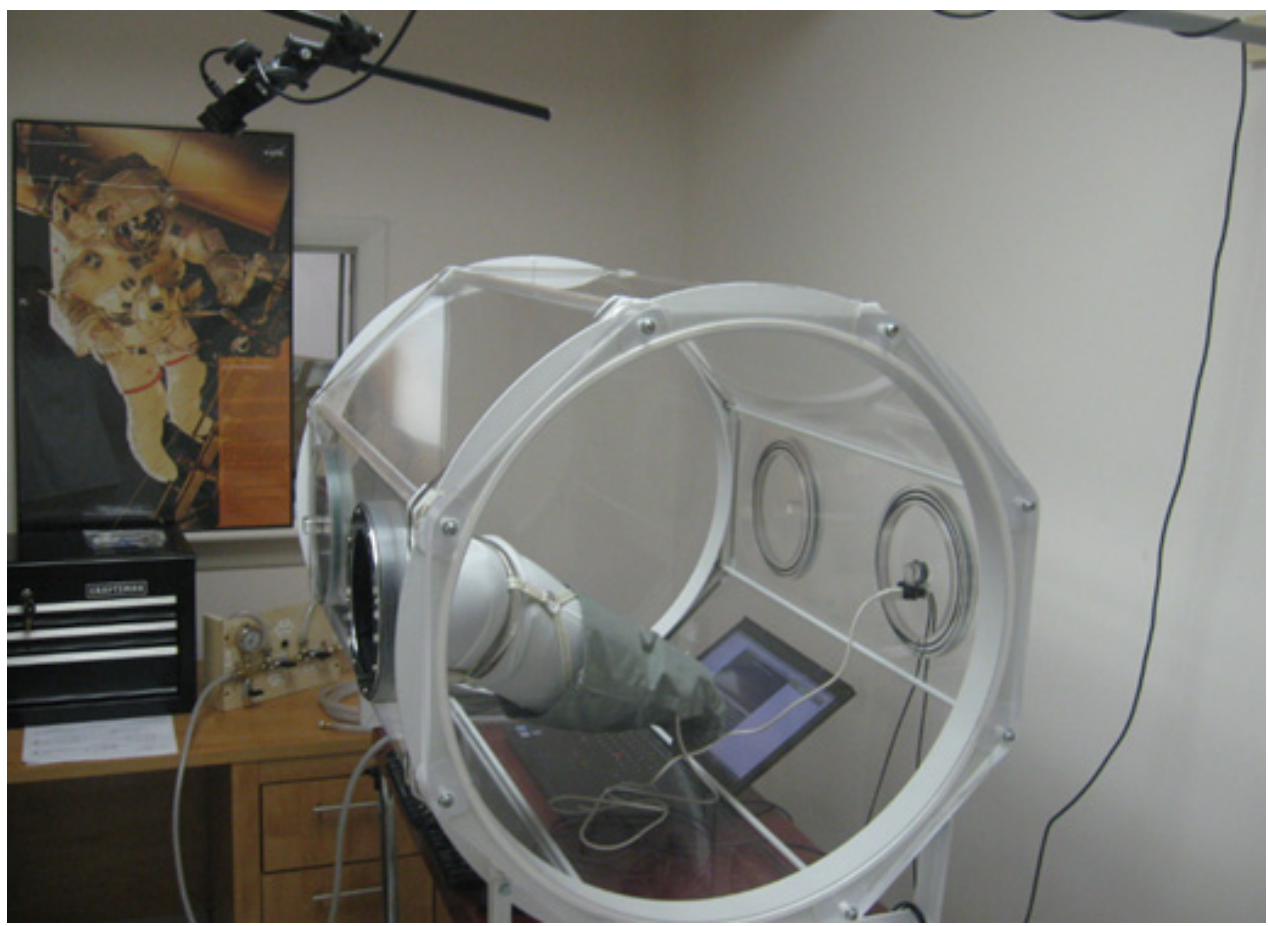

Figure 5.-GECO test setup.
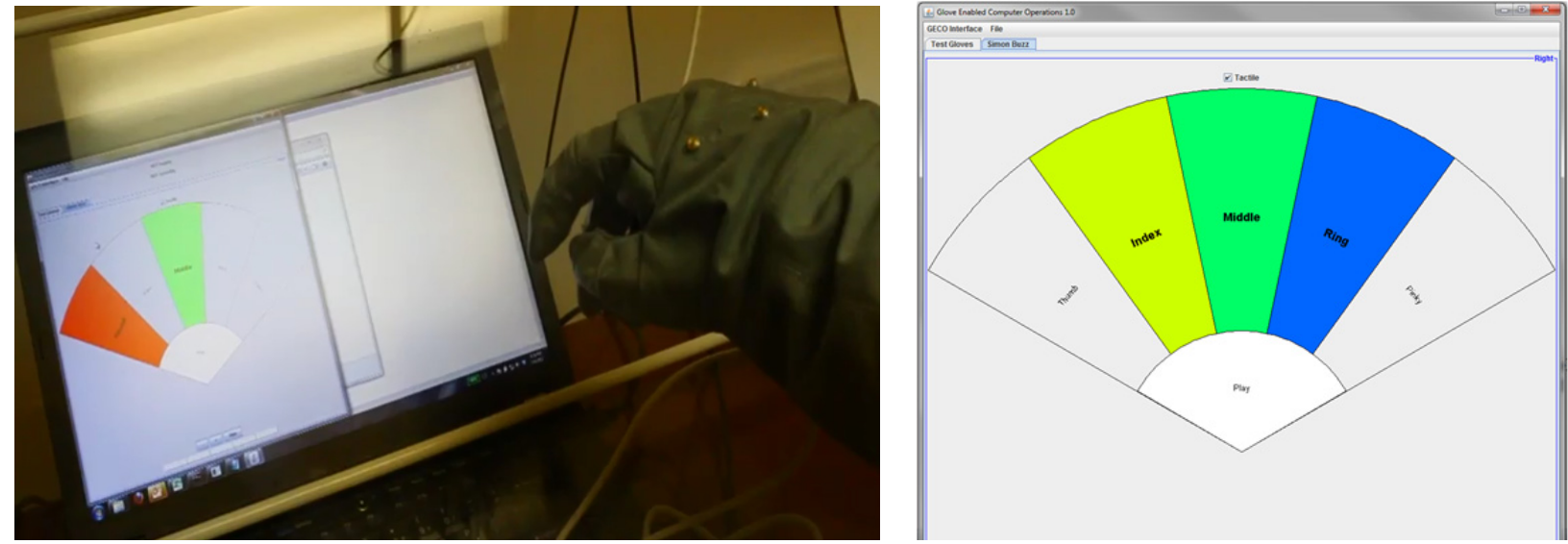

Figure 6.- Simon says game.

To facilitate testing, Barron Associates developed a suite of software applications, including games and automated data entry tasks, which challenge a user's ability to employ the GECO gloves as a data entry device. These include (1) a Simon Says Game, inspired by the electronic toy game, in which users attempt to follow cues for randomly-generated sequences of finger taps; and (2) Virtual Keyboard Data Entry, in which users employ the GECO glove in both MOUSE and KEYBOARD modes to enter randomly-generated word sequences using an on-screen virtual keyboard.

The Simon Says Game was constructed as a simple, and familiar, activity to assess a user's ability to correctly reproduce randomly-generated finger strike sequences (Figure 6). The computer plays the role of "Simon," starting with a single target finger, and then progressively adding randomly-generated target fingers with each subsequent sequence. The target sequence is first presented to the user through visual, audio (unique tone), and finger-specific tactile feedback. The Simon Game was configured such that it can be played in two alternate conditions: non-tactile and tactile. In the tactile condition, the user receives localized (finger specific) tactile feedback indicating a finger strike has been registered. No visual or 

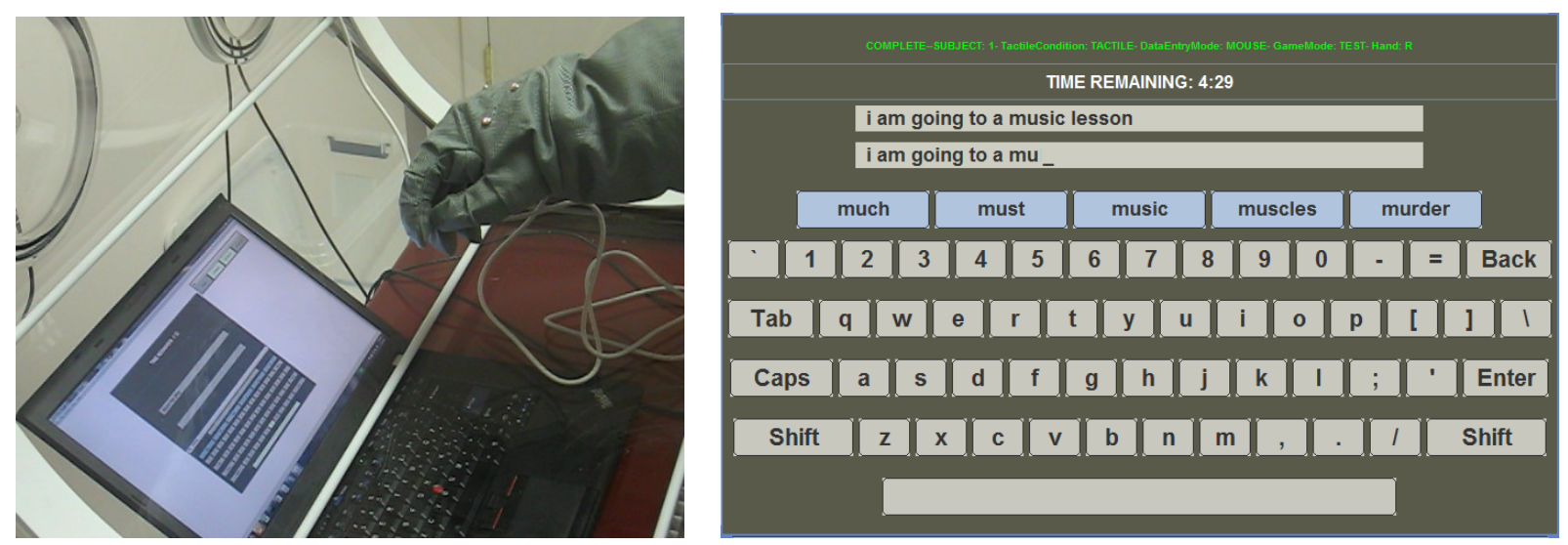

Figure 7.-Text entry activity.

audio cues are provided for the user's finger movement. If a mistake is made (i.e., registered finger does not match the target), the sequence is terminated and the game moves on to the subsequent sequence. In every case, the game proceeds until five sequences have been completed, resulting in up to fifteen total finger strikes per game.

The Text Entry activity was designed around a Java-based virtual keyboard application that automates human subject data collection and generation of text entry performance measures (Figure 7). The virtual keyboard can be employed in a "hunt-and-peck" or "virtual touch typing" condition by placing the GECO glove in MOUSE or KEYBOARD mode, respectively. The virtual keyboard can also be employed with or without vibrotactile feedback, by activating or deactivating the glove actuators. During test execution, the software presents a target phrase randomly drawn from an internal database. The user employs the virtual keyboard to transcribe the phrase as quickly and accurately as possible. When entered, a new phrase appears, the cycle continuing for the duration of the test. On completion, software generates the three performance metrics: CPM (the average number of correct characters entered per minute), KSC (average number of keystrokes per correct character entered), and MSD (average mean string distance between the targeted and user-entered phrases) using published string processing algorithms (Ref. 13).

\subsection{Procedures and Statistical Design}

On arrival, volunteers received a pre-test briefing and overview of the NASA IRB-approved consent form. Test procedures were initiated upon provision of consent. The participant's right hand was traced employing a standard template and size verified to be within the pre-established ranges. Participants completed the Simon Game activity in an initial visit and performed the Text Entry activities in a second visit.

\subsubsection{Simon Game}

Participants initially played four Simon Games for practice, half with and half without tactile feedback. They then played 20 games for record, again alternating between tactile conditions. The within subjects protocol design, evaluating each subject under both conditions, was motivated by anticipated high inter-subject variability in performance. The alternating presentation of conditions between games was introduced in anticipation of a significant training effect (Ref. 11). Test software automatically recorded the cued and user-entered finger strikes into a comma-separated-value file for subsequent statistical analysis.

The primary objective of Simon Game data analysis was to determine whether participants' accuracy in generating finger strikes was enhanced by tactile feedback. A secondary objective was to assess the expected overall accuracy participants may achieve in generating finger strikes. Although each game was played to a length of five sequential finger strikes, only the first three sequences in any game were 
included in the primary analysis to reduce the potential impact of memory-related errors on the outcome. This decision was based on the experience of previous research into working memory and sequence learning ability (Ref. 14). Additional objectives included assessing the association between accuracy and the targeted finger (thumb through little finger). Another was to examine whether there was an association between accuracy and the length of the targeted sequence (working memory/sequence learning effect). The final secondary objective was to examine if there was an association between accuracy and the number of games played (learning, or alternatively, fatigue effects).

The Simon Game outcome data were analyzed by way of a binomial generalized estimating equation model (GEEM) (Ref. 15), to evaluate average performance; and a binomial generalized linear mixed model (GLMM) (Ref. 16), to evaluate subject-specific performance. An a priori $\mathrm{p} \leq 0.05$ decision rule was established as the null hypothesis rejection criterion. The GEEM had a single predictor variable that distinguished between the tactile feedback trials and the non-tactile feedback trials. Since a participant's game performance from trial to trial could not be assumed to be statistically independent, the Huber "sandwich" estimator was utilized to estimate the variance-components of the GEEM variance-covariance matrix. The GEEM link function was the logit,

$$
\log \left(\frac{\pi_{i j}}{1-\pi_{i j}}\right)=\beta+\beta_{1} I_{i j}(\text { Tactile Feedback }=\text { No })
$$

where $\pi_{i j}$ is the probability that subject $i$ finger of response on trial $j$ was the target finger.

The Wald chi-square test was used to test the null hypothesis that the odds of a target finger match (i.e., the finger of motion matched the target finger) is the same irrespective of tactile condition. $95 \%$ confidence intervals were constructed to estimate the percentage of trials in which the finger of motion matched the target finger for each experimental condition. GEEM analyses were conducted using the GENMOD procedure of SAS software version 9.2 (SAS Institute Inc., Cary, NC).

In evaluation of subject-specific performance, the GLMM fixed-effect was the experimental condition (tactile feedback versus no tactile feedback), and GLMM random-effect was the subject number. GLMM parameter estimation was based on the principle of restricted maximum likelihood and the GLMM link function was the logit,

$$
\log \left(\frac{\pi_{i j}}{1-\pi_{i j}}\right)=\beta+\beta_{1} I_{i j}(\text { Tactile Feedback }=\text { No })+b_{1 i}
$$

where $b_{1 i}$ is the random effect associated with subject $i$.

Construction of $95 \%$ prediction intervals was based on the normal approximation method. GLMM analyses were conducted using the GLIMMIX procedure of SAS software version 9.2. At the end of the Simon Game session, subjects completed the NASA TLX to provide a subjective assessment of user workload (Mental Demand, Physical Demand, Temporal Demand, Performance, Effort, and Frustration) (Ref. 17). The primary objective of the Simon Game survey analyses was to compare participants' opinions about the mental, physical, and temporal demands; as well as game performance, effort, and level of frustration when playing the Simon Game with and without tactile feedback. The response to each question was measured on a 20-point Likert-scale with higher points reflecting greater demand, greater performance, greater effort, and greater frustration. The integer-scale responses to each survey question were analyzed by way of a negative binomial generalized linear model (GLM). The GLM dependent variable was the integer-scale response to the question, and the independent variable identified whether response was in reference to the tactile feedback game trails, or in reference to the non-tactile feedback game trials. The GLM parameters were estimated by way of maximum likelihood and the GLM link function was the natural logarithm. 
For each survey question, the Wald chi-square test was used to test the null hypothesis that the mean response was the same regardless of whether the question was in reference to the tactile feedback game trials, or in reference to the non-tactile feedback game trials. A $\mathrm{P} \leq 0.05$ decision rule was established $a$ priori as the null hypothesis rejection criterion. 95\% confidence interval construction for the mean response, and for the ratio of mean responses, was based on the Wald method. The software of the GENMOD procedure of SAS 9.2 was used to conduct the survey questionnaire analyses.

\subsubsection{Text Entry Activity}

In a within-subjects design, each participant performed the Text Entry activity for $5 \mathrm{~min}$ in both hunt-and-peck and touch-typing modes, both with and without tactile feedback (for a total of 10 min of recorded text entry in each feedback condition). Prior to each timed test event, participants first practiced in the given combination of text entry and tactile mode for $5 \mathrm{~min}$. The primary objective of the data analysis was to determine if the participant's typing performance was enhanced by tactile feedback. As secondary objectives, we examined if typing performance was dependent upon the mode of typing, and if typing performance was dependent upon trial order (training effect). The primary performance measure was the number of characters typed per minutes (CPM) over a 5-min trial. Secondary error measures included keystrokes per correct character entered (KPC) and mean string distance between target phrases and entered text (MSD).

CPM and MSD data were analyzed by way of a negative binomial generalized estimating equation model, and the KPC data were analyzed by way of a Poisson generalized estimating equation model. The dependent variable for the CPM analysis was the total number of correct characters typed during the 5 min typing performance trial. The dependent variable for the KPC analysis was the total number of key strokes (correct and incorrect) entered, and the dependent variable for the MSD analysis was the total string length between entered versus targeted phrases. Each GEEM had three predictor variables. One variable distinguished the tactile feedback typing performance trials from the non-tactile feedback typing performance trials. One variable distinguished the touch-typing condition from hunt-and-peck, and the remaining predictor variable was tactile feedback mode by typing mode interaction. Since a participant's performance from trial to trial could not be assumed to be statistically independent, the Huber "sandwich" estimator was utilized to estimate the variance-components of the GEEM variance-covariance matrix. The natural logarithmic link function was used for both the negative binomial GEEM and the Poisson GEEM. The GEEM offset variable for the CPM analysis was the time duration of the typing performance trial (i.e., $5 \mathrm{~min}$ ). The GEEM offset variable for the KPC analysis was the number of correct characters entered, and the GEEM offset variable for MSD was the total number of target characters.

With regard to hypothesis testing, the GEEM version of the Wald chi-square test was used to test the 2 primary null hypotheses: (1) that the mean of the distribution of the performance measurements for the touch-typing performance trials is the same regardless of whether or not the typing performance trial is performed with the aid of tactile feedback, and (2) that the mean of the distribution of the performance measurements for the hunt-and-peck performance trials is the same regardless of whether or not the typing performance trial is performed with the aid of tactile feedback. A $p \leq 0.05$ decision rule was established a priori as the null hypothesis rejection criterion. The following two secondary hypotheses were also examined: (1) the mean of the distribution of the performance measurements for the with-tactile feedback performance trials is the same regardless of whether or not the trials are performed via touchtyping or hunt-and-peck method, and (2) the mean of the distribution of the performance measurements for the without-tactile feedback typing performance trials is the same regardless of whether or not the trials are performed via touch-typing or hunt-and-peck method. Hypotheses directed at testing for tactile mode and typing mode main effects were also performed when the test for tactile mode by typing-mode interaction was not rejected at $p \leq 0.05$. A $p \leq 0.05$ decision rule was established a priori as the null hypothesis rejection criterion for the secondary hypothesis tests and for the hypotheses tests related to main effect. 95\% confidence interval construction for estimating the ratio of mean performance was based the GEE version of the Wald normal approximation method. 
At the end of the Text Entry activities, subjects completed the NASA TLX instrument to provide a subjective assessment of user workload (Mental Demand, Physical Demand, Temporal Demand, Performance). The Text Entry TLX data were analyzed in a like fashion to the Simon Game TLX data.

\subsection{Results}

\subsubsection{Simon Game Results}

Over all subjects and trials, with tactile feedback, correct finger strikes were made 1143 times while incorrect finger strikes were made 33 times. Without tactile feedback, the corresponding totals were 1133 and 40. Mean values and confidence intervals generated using the aforementioned GEM model are shown in Figure 8. The finger of motion matched the target finger 97.2\% of the time (95\% CI: [95.9, 98.1\%]) with tactile feedback, and $96.6 \%$ of the time (95\% CI: [95.1, 97.6\%]) without $(\mathrm{p}=0.466)$.

The subject-specific predictions for the mean percentage of target finger matches, generated using the GLMM model, are shown in Figure 9. Results are ordered by increasing mean. Subject-specific predictions range from 90.1 to $99.3 \%$ for the tactile feedback trials and from 88.2 to $99.1 \%$ for the nontactile trials.

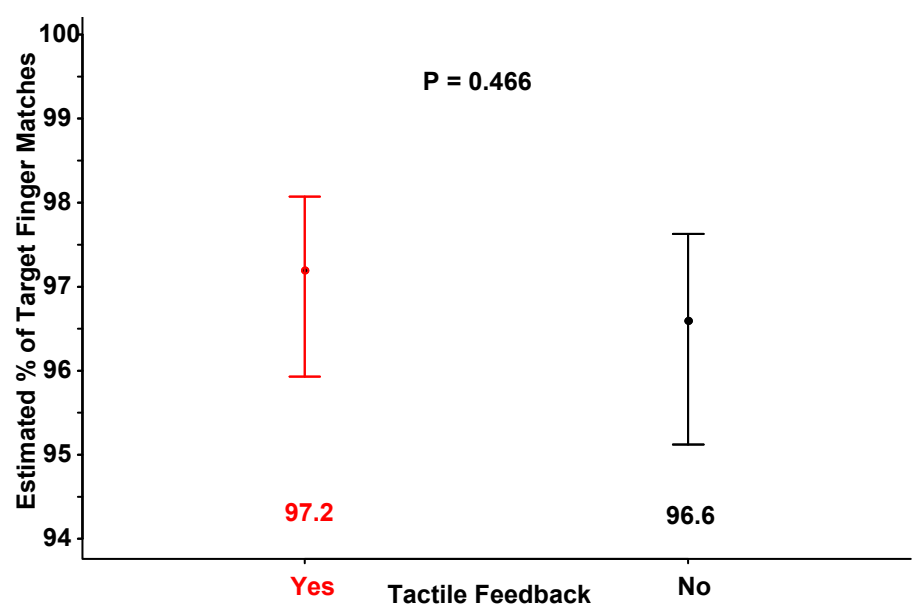

Figure 8.-Target finger matches versus tactile condition.

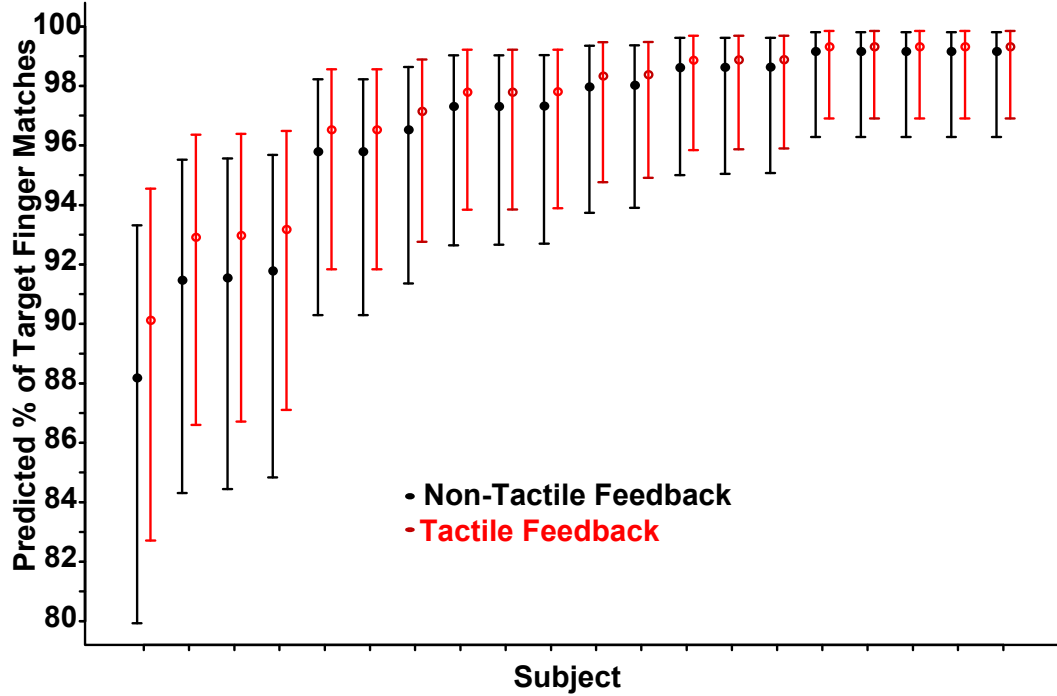

Figure 9.-Subject specific predictions. 
The concordance between the target finger and the finger of motion is summarized in Table 1 . The percentage of mismatches associated with a given target finger are shown in parentheses. For all trials (i.e., irrespective of tactile feedback status), 61.9\% (95\% CI: [55.6, 68.0\%]) of finger mismatches involved an adjacent finger. For the tactile feedback trials, 66.4\% (95\% CI: [56.9, 75.0]) of finger mismatches involved an adjacent finger, and for the non-tactile feedback trials 58.2\% (95\% CI: [49.4, 66.7]) of finger mismatches involved an adjacent finger.

For the tactile feedback Simon Game trials, when the target finger was either the thumb, index finger, or middle finger, the percentage of target finger matches was $97.3 \%$ (95\% CI: [96.2, 98.1\%]), whereas, when the target finger was either the ring finger or little finger, the percentage of target finger matches was 94.1\% (95\% CI: [92.0, 95.7\%]) $(\mathrm{p}<0.001)$ (Figure 10). For the non-tactile feedback Simon Game trials, the corresponding results are 96.8 (95\% CI: [95.7, 97.7]) for thumb-index-middle and $92.9 \%$ (95\% CI: [90.7, 94.6\%]) $(\mathrm{p}<0.001)$ for ring-little finger. The impact of the finger composition on the percentage of target finger matches was unrelated to tactile condition.

\section{TABLE 1.-CONCORDANCE-TARGET AND FINGER OF MOTION}

\section{Finger of Motion}

\begin{tabular}{|c|c|c|c|c|c|}
\hline Target Finger & thumb & index & middle & ring & little \\
\hline thumb & 1016 & $18(46.1)$ & $18(46.1)$ & $2(5.1)$ & $1(2.5)$ \\
\hline index & $9(22.5)$ & 1116 & $22(55.0)$ & $3(7.5)$ & $6(15.0)$ \\
\hline middle & $6(30.0)$ & $7(35.0)$ & 1138 & $4(20.0)$ & $3(15.0)$ \\
\hline ring & $0(0)$ & $15(20.3)$ & $57(77.0)$ & 1096 & $2(2.7)$ \\
\hline little & $13(17.6)$ & $13(17.6)$ & 14 (18.9) & 34 (45.9) & 1045 \\
\hline
\end{tabular}

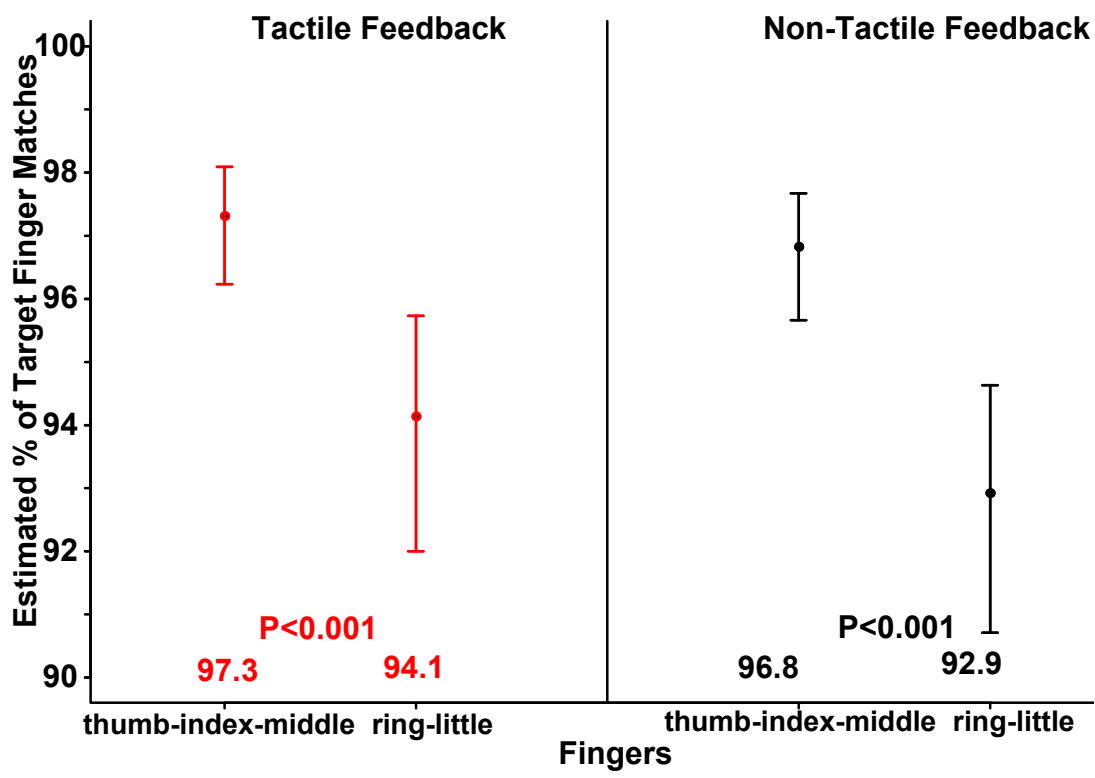

Figure 10.-Impact of target finger. 


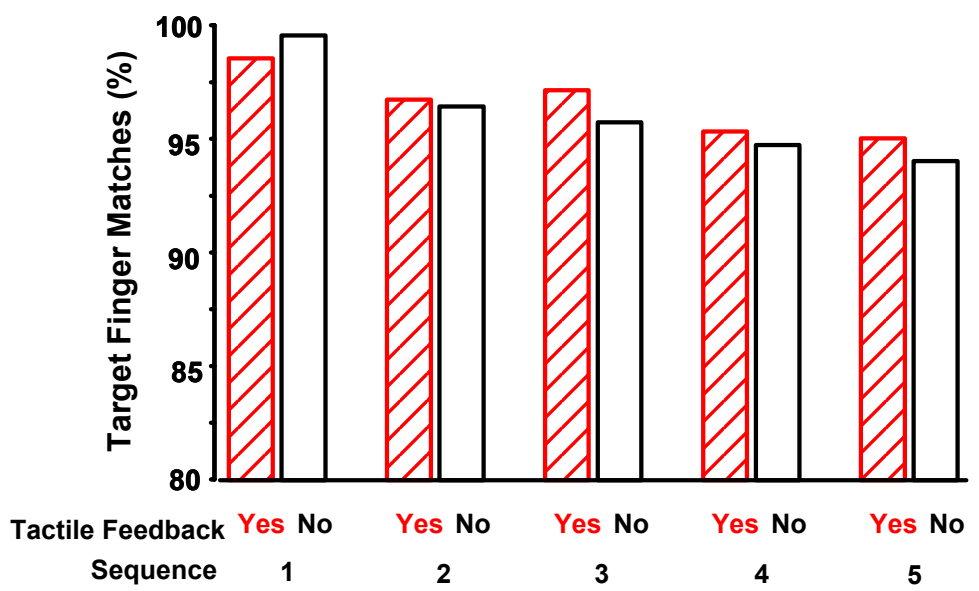

Figure 11.-Match frequencies versus target sequence length.

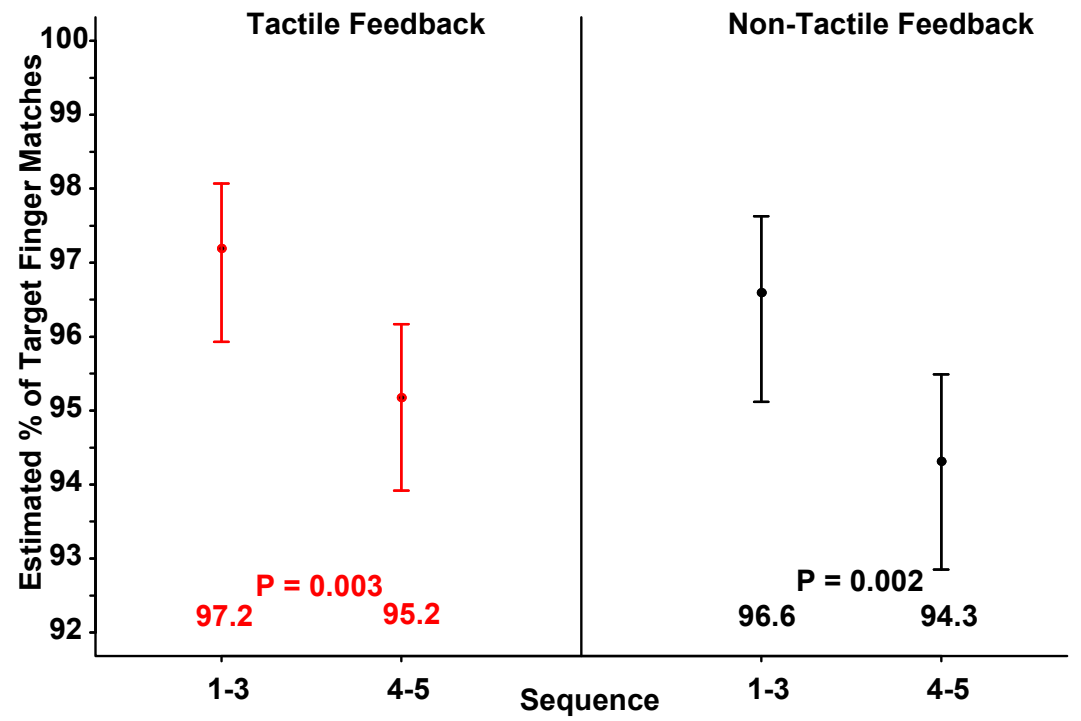

Figure 12.-Impact of target sequence length.

Frequencies for the number of target finger matches and mismatches as a function of game sequence number and tactile condition are summarized in Figure 11.

Predictions for the impact of sequence length on predicted performance based on an extended GEEM model are shown in Figure 12. For the tactile condition, when the target sequence had a length of 3 or fewer finger strikes, the percentage of target finger matches was $97.2 \%$ (95\% CI: [95.9, 98.1\%]), whereas, when the sequence had a length of 4 or 5, the percentage of target finger matches was $95.2 \%$ (95\% CI: $[93.9,96.2 \%])(\mathrm{p}=0.003)$. For the Simon Game in the non-tactile condition, when the sequence had a length of 3 or fewer, the percentage of target finger matches was $96.6 \%(95 \%$ CI: [95.9, 98.1\%]), whereas, when the sequence had a length of 4 or 5 , the percentage of target finger matches was $94.3 \%$ $(95 \%$ CI: $[92.9,95.5 \%])(\mathrm{p}=0.002)$. The impact of the sequence length on the percentage of target finger matches was unrelated to tactile condition $(\mathrm{p}=0.907)$.

As described above, each subject played 20 Simon games in total, ten in each condition, alternating between conditions with each game. The relationship between the percentage of target finger matches and the sequential game number (i.e., game 1 was played first, game 20 last) is summarized in tabular form in Figure 13. No significant association (represented as the slope regression parameter in a generalized estimating model) was found between the odds of target match and the game number ( $p=0.883$, and $p=0.204$, respectively) in either the tactile feedback or non-tactile feedback trials. 


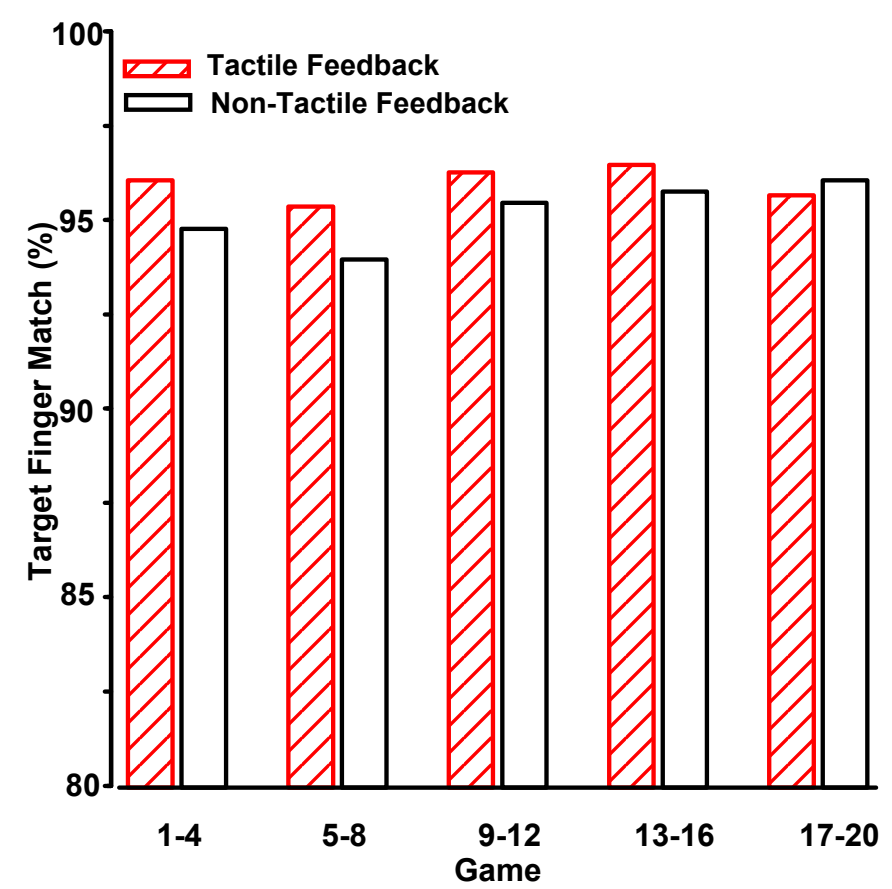

Figure 13.-Match frequencies versus game number.

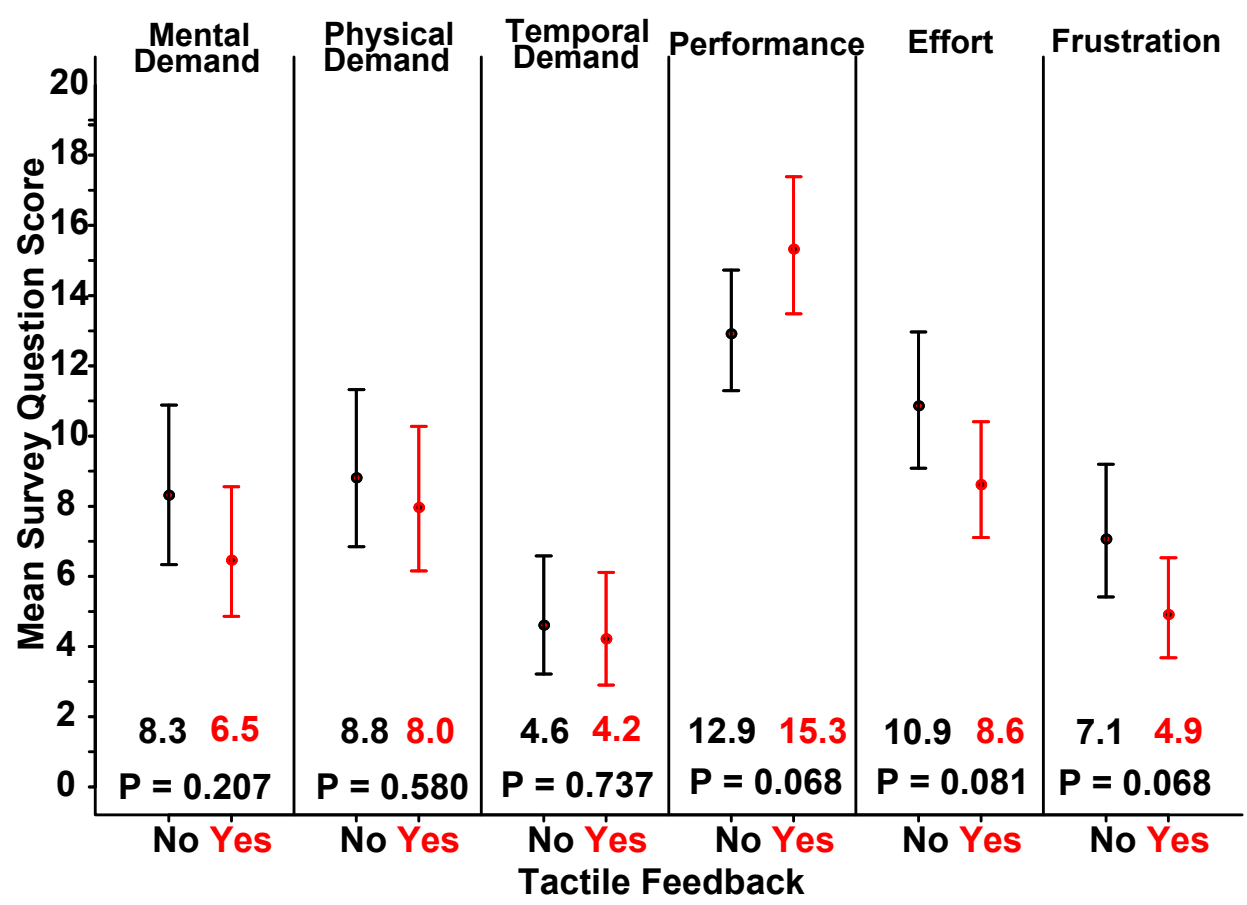

Figure 14.-NASA TLX analysis summary.

The distributions of Likert-scale questionnaire responses from the NASA TLX survey are summarized in Figure 14. The mean scores for "mental demand" were 6.5 (95\% CI: [4.9, 8.6]) and 8.3 (95\% CI: $[6.3,10.9])$, for the tactile feedback and the non-tactile feedback surveys questions, respectively $(\mathrm{p}=0.207)$. The mean scores for "physical demand" were 8.0 (95\% CI: $[6.2,10.3])$ and 8.8 $(95 \%$ CI: $[6.8,11.3]),(\mathrm{p}=0.580)$, and the mean scores for "temporal demand" were 4.2 (95\% CI: [2.9, $6.1])$ and $4.6(95 \% \mathrm{CI}[3.2,6.6]),(\mathrm{p}=0.737)$. 
The mean scores for "performance" were 15.3 (95\% CI $[13.5,17.4])$ and 12.9 (95\% CI [11.3, 14.7]), respectively, for the tactile feedback and the non-tactile feedback survey questions $(\mathrm{p}=0.068)$. The mean scores for "effort" were $8.6(95 \%$ CI $[7.1,10.4])$ and 10.9 (95\% CI $[9.1,13.0]),(p=0.081)$; and the mean scores for "frustration" were 4.9 (95\% CI [3.7, 6.5]) and 7.1 (95\% CI [5.4, 9.2]), $(\mathrm{p}=0.068)$.

The mean for the composite of the objective task demand-related (Ref. 17) responses (the sum of the "mental", "physical", and "temporal" demand scores) for the tactile feedback and the non-tactile feedback survey questions were 17.8 (95\% CI: [4.3, 22.1]) and 21.7 (95\% CI: [17.7, 26.7]), respectively ( $\mathrm{p}=$ 0.192). The mean for the composite of the subjective behavioral-related responses (Ref. 17) of "effort" and "frustration" were 13.5 (95\% CI: [11.2, 16.3]) with tactile feedback and 17.9 (95\% CI: [14.9, 21.3]), without, $(p=0.035)$.

\subsubsection{Text Entry Activity Results}

The empirical distributions of the CPM measurements across all subjects are shown in Figure 15.

For the virtual touch typing trials, the mean of the CPM measurement distribution was 28.8 (95\% CI: $[26.0,32.0])$ with tactile feedback, and $25.4(95 \% \mathrm{CI}$ : $[22.5,28.6])$ without tactile feedback $(\mathrm{p}=0.005)$ (Figure 16(a)). For the hunt-and-peck typing trials, the mean of the CPM measurement distribution was 37.5 (95\% CI: [35.7, 39.4]) with tactile feedback, and 35.9 (95\% CI: [34.2, 37.7]) without tactile feedback $(\mathrm{p}=0.065)$. Across both data entry modes, the mean of the CPM distribution was $8 \%$ (95\% CI: $[3,13 \%])$ greater with tactile feedback than without $(p=0.001)$ (Figure 17).

For performance trials performed with tactile feedback, the mean of the CPM distribution was $30 \%$ (95\% CI: [19, 41\%]) greater in hunt-and-peck mode versus touch-typing $(\mathrm{p}<0.001)$ (Figure 16(b)). Without tactile feedback, the mean of the CPM distribution was $41 \%$ (95\% CI: [29, 56\%]) greater in hunt-and-peck mode versus touch-typing $(\mathrm{p}<0.001)$. Across tactile feedback conditions, the mean of the CPM distribution was 35\% (95\% CI: [25, 46\%]) greater in hunt-and-peck mode versus touch-typing $(p<0.001)$ (Figure 17).

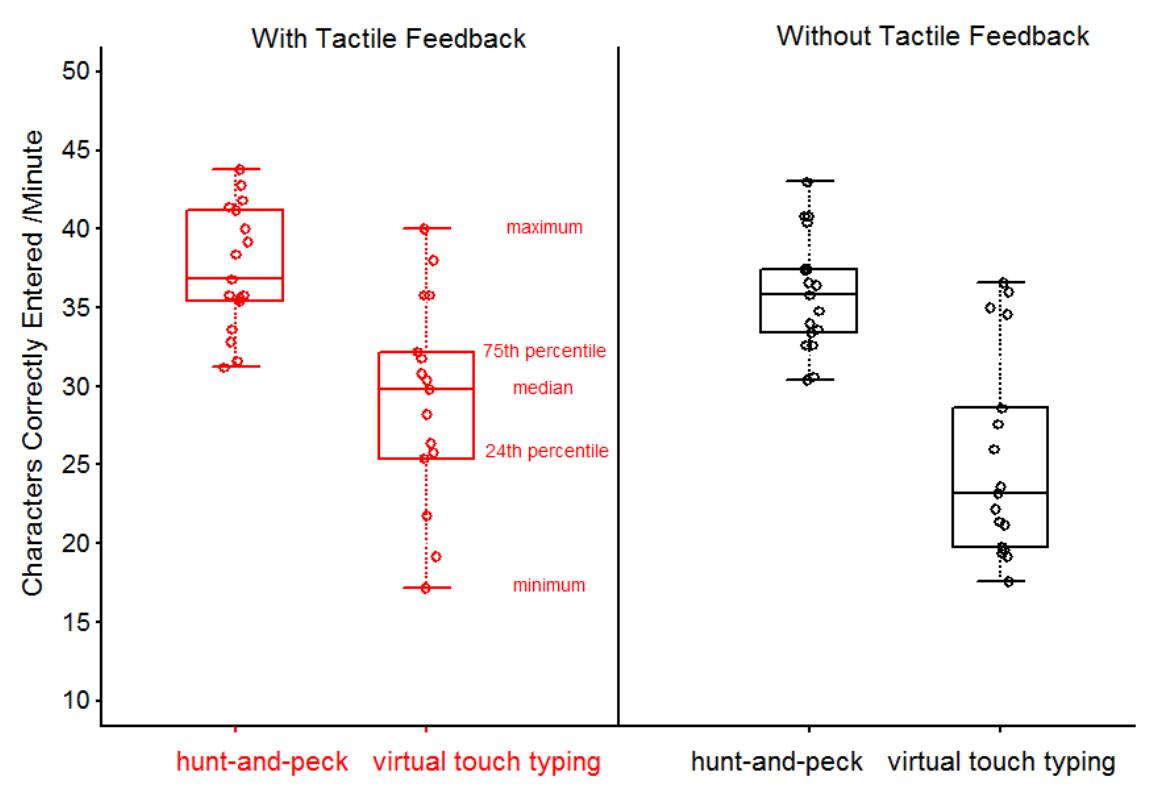

Figure 15.-Characters per minute (CPM) data summary. 


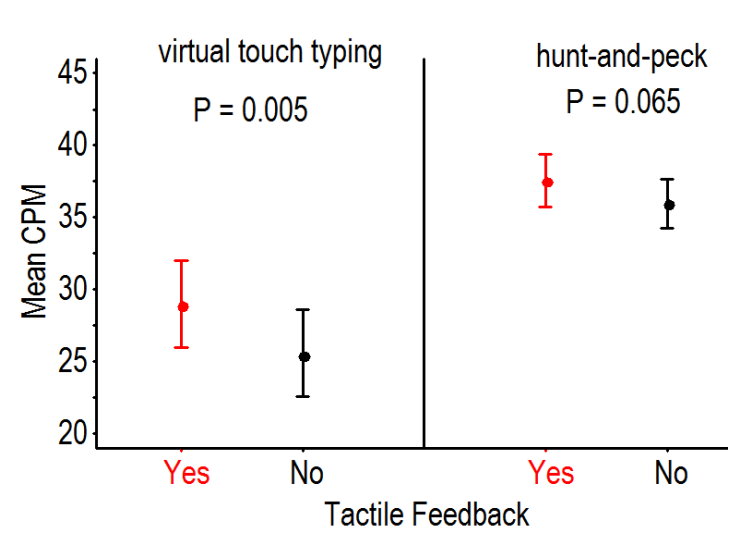

(a) Effect of Tactile Feedback

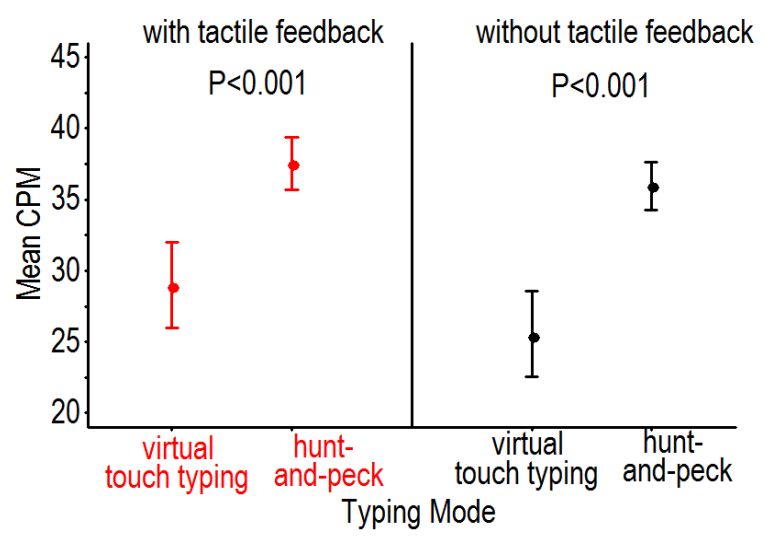

(b) Effect of Typing Mode

Figure 16.-Characters per minute (CPM) analysis.

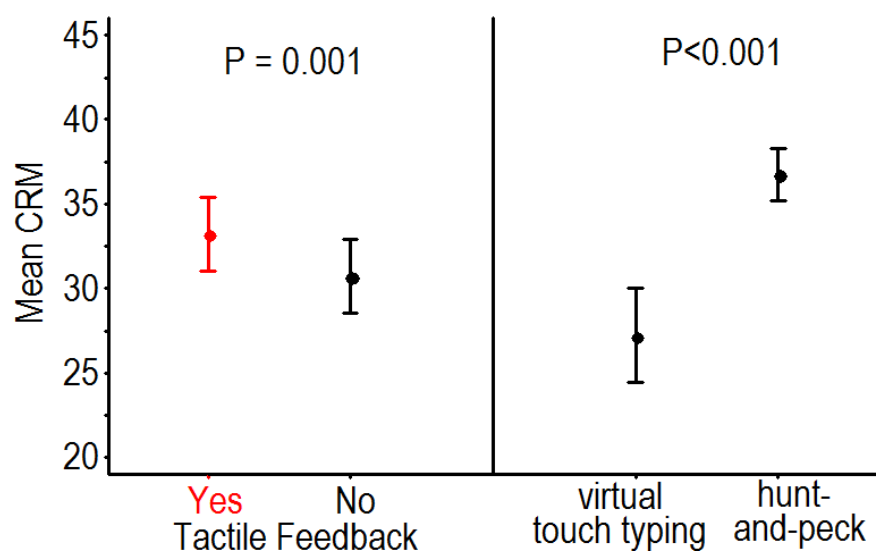

Figure 17.-Characters per minute (CPM) marginal (average) effect summary. 
The empirical distributions of the KPC measurements across all subjects are shown in Figure 18. For the touch-typing performance trials (Figure 19(a)), the mean of the KPC measurement distribution was 1.07 (95\% CI: [1.04, 1.10]) with tactile feedback and 1.10 (95\% CI: [1.07, 1.14]) without $(\mathrm{p}=0.054)$. For the hunt-and-peck trials, the mean of the KPC measurement distribution was 1.02 (95\% CI: [1.01, 1.02]) with tactile feedback and 1.02 (95\% CI: [1.01, 1.03]) without $(\mathrm{p}=0.319)$. Across data entry modes, the mean of the KPC distribution was $2 \%(95 \% \mathrm{CI}:[0.0,3 \%])$ less for with tactile than without $(\mathrm{p}=0.023)$ (Figure 20).

For performance trials with tactile feedback, the mean of the KPC distribution was 5\% (95\% CI: $[2,7 \%])$ less when the hunt-and-peck typing method was used compared to touch-typing $(\mathrm{p}<0.001)$

(Figure 19(b)). For performance trials without tactile feedback, the mean of the KPC distribution was 7\% $(95 \%$ CI: $[5,10 \%])$ less in hunt-and-peck mode versus touch-typing $(\mathrm{p}=0.001)$. Across both tactile modes, the mean of the KPC distribution was 6\% (95\% CI: [4, 8\%]) less hunt-and-peck compared to touch-typing $(\mathrm{p}<0.001)$ (Figure 20).

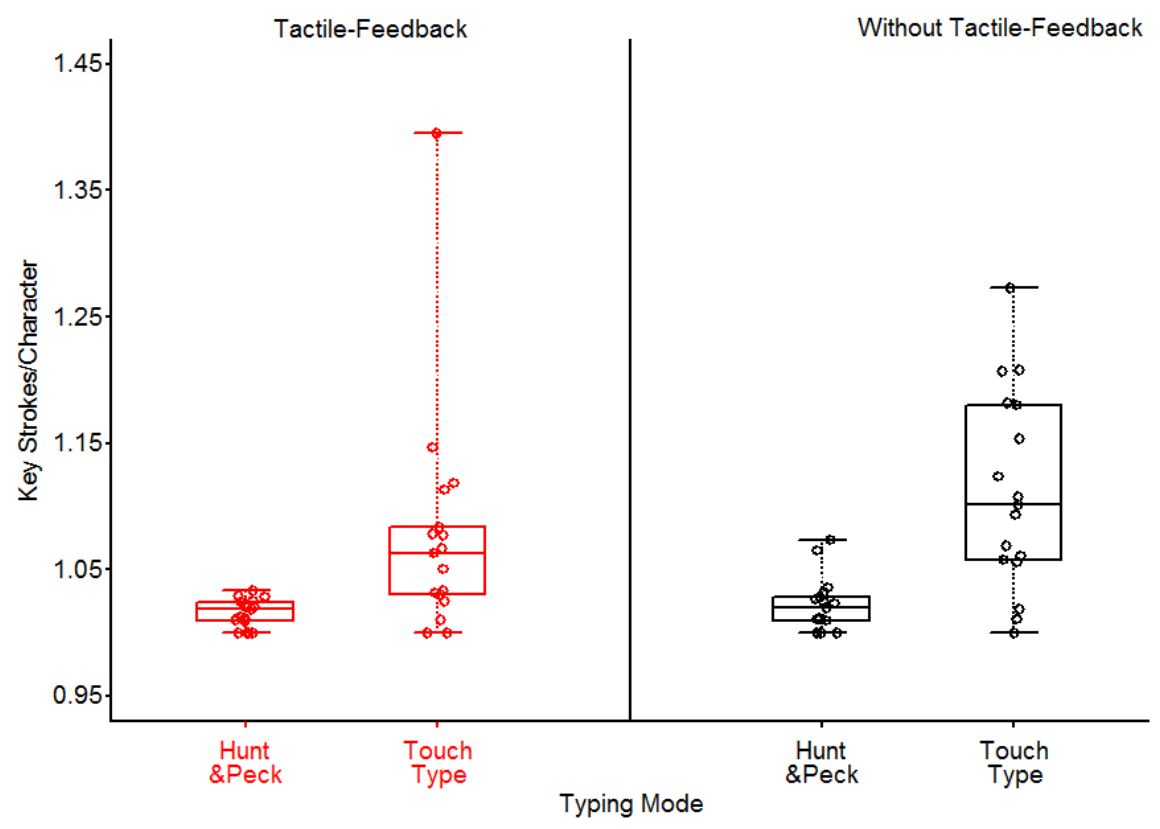

Figure 18.-Keystrokes per character (KPC) data summary.

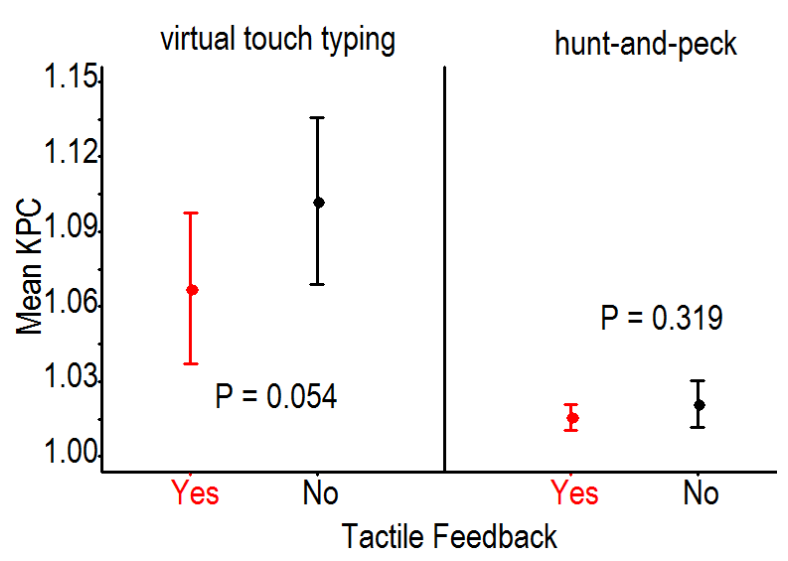

(a) Effect of Tactile Feedback

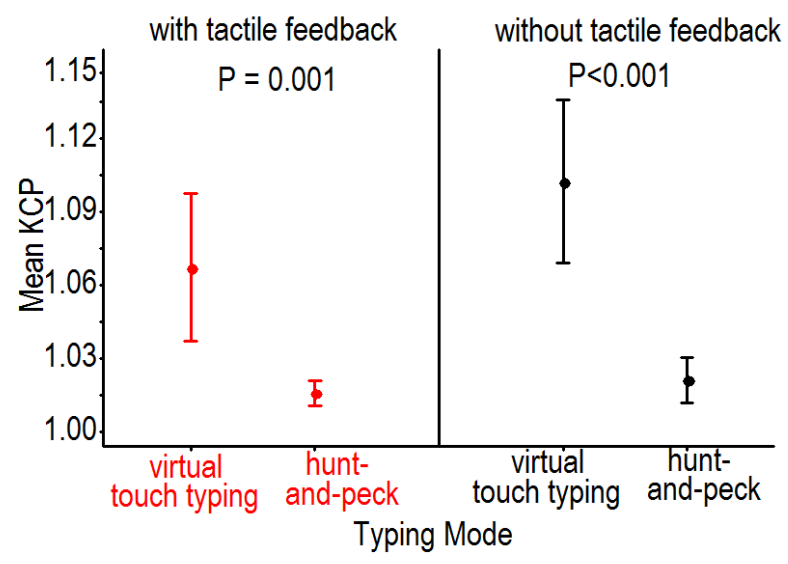

(b) Effect of Typing Mode

Figure 19.-Keystrokes per character (KPC) analysis. 

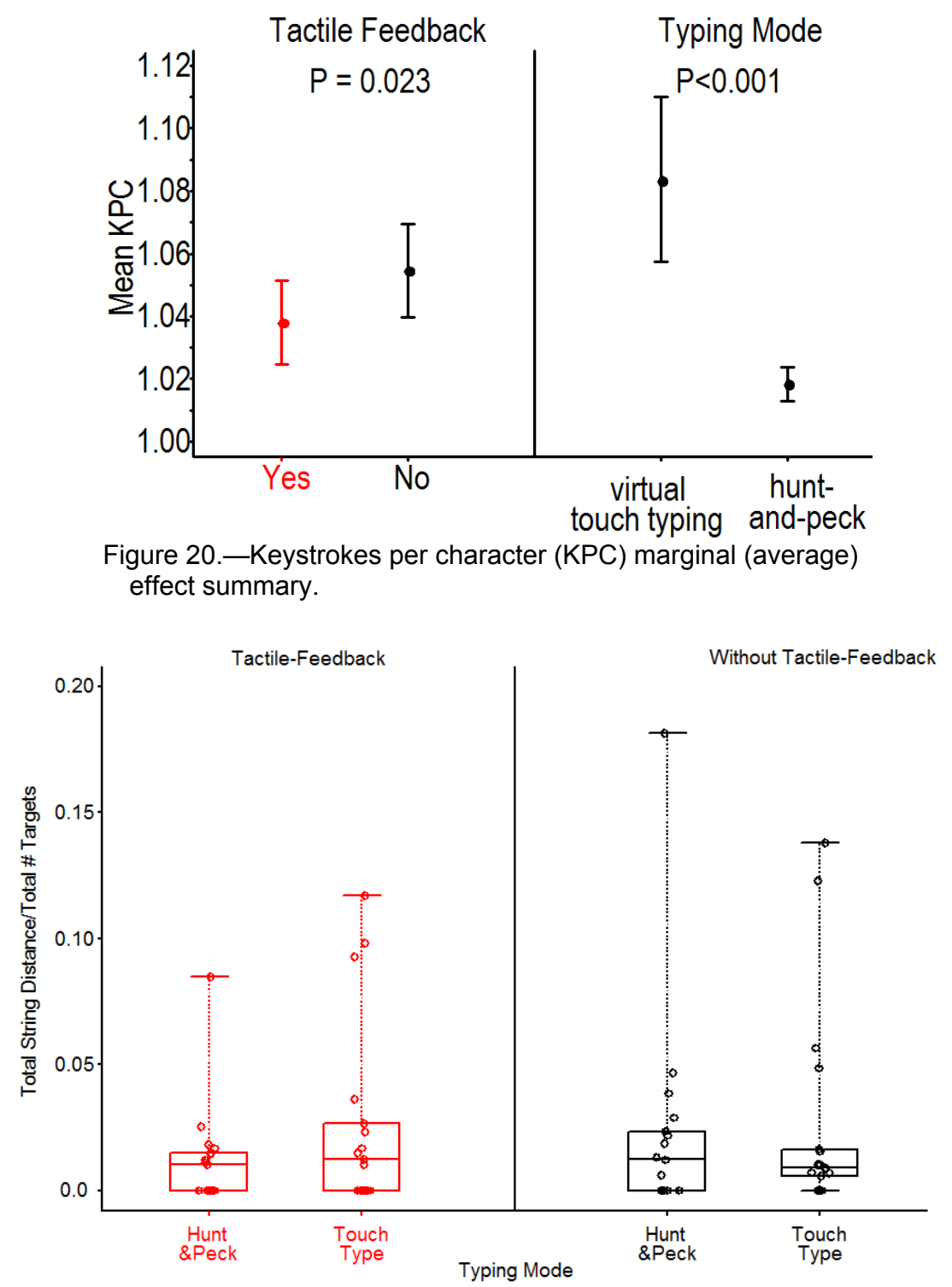

Figure 21.-Mean string distance (MSD) data summary.

The empirical distributions of the MSD measurements across all subjects are shown in Figure 21.

For the touch-typing performance trials, the mean of the MSD measurement distribution was 0.026 (95\% CI: [0.013, 0.051]) with tactile feedback compared to 0.026 (95\% CI: [0.013, 0.054]) without $(\mathrm{p}=0.992)$ (Figure 22(a)). For the hunt-and-peck trials, the mean of the MSD measurement distribution was 0.012 (95\% CI: [0.006, 0.027]) with tactile feedback compared to 0.023 (95\% CI: [0.010, 0.056]) without $(\mathrm{p}=0.002)$. As there was significant tactile condition by typing condition interaction $(\mathrm{p}=0.038)$, it is inappropriate to estimate the marginal (average) effect of tactile feedback across data entry conditions.

For performance trials with tactile feedback, the mean of the MSD distribution was $53 \%(95 \% \mathrm{CI}$ : $[3,77 \%])$ less for hunt-and-peck versus touch-typing $(\mathrm{p}=0.042)$ (Figure 22(b)). Without tactile feedback, the mean of the MSD distribution was $12 \%$ (95\% CI: [-86, 58\%]) less for hunt-and-peck versus touchtyping $(\mathrm{p}=0.737)$. As there was significant tactile condition by typing condition interaction $(\mathrm{p}=0.038)$, it is inappropriate to estimate the marginal (average) effect of data entry mode across tactile condition. The GEE regression analyses show that trial order (order in which tactile feedback was not associated with the primary performance measure CPM $(\mathrm{p}=0.528)$, nor was "trial order" associated with KPC $(\mathrm{p}=0.307)$, or $\operatorname{MSD}(\mathrm{p}=0.177)$. 


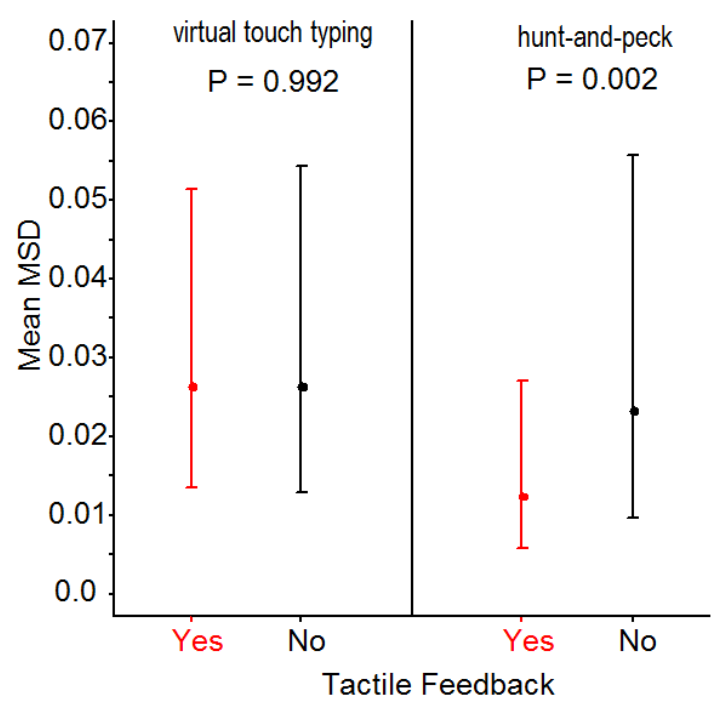

(a) Effect of Tactile Feedback

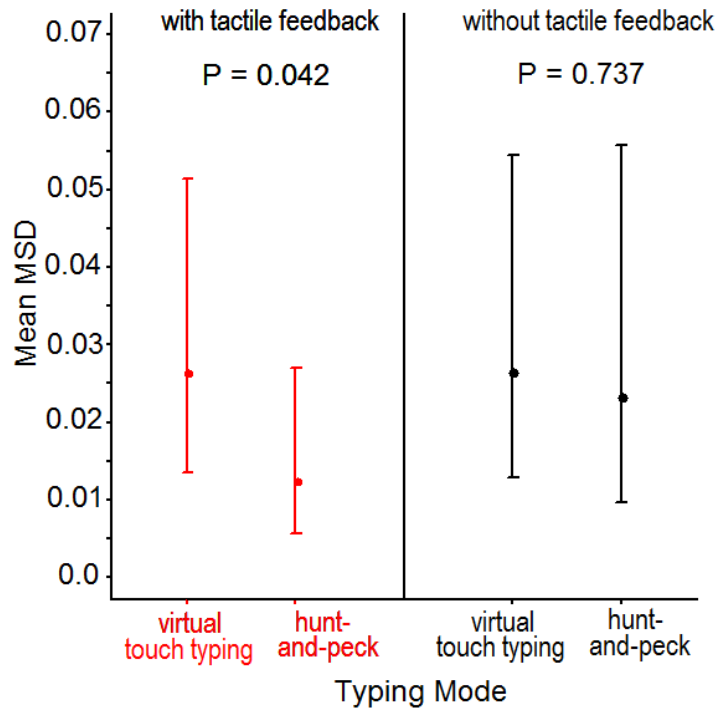

(b) Effect of Typing Mode

Figure 22.-Mean string distance (MSD) analysis.

The confidence intervals and means values of the NASA TLX categories for the hand-and-peck trials are summarized in Figure 23. The mean scores for "mental demand" were 6.3 (95\% CI: [4.8, 8.4]) with tactile feedback versus $6.7(95 \% \mathrm{CI}:[5.3,8.6])$ without $(\mathrm{p}=0.150)$. The mean scores for "physical demand" were 13.5 (95\% CI: [12.0, 15.2]) and 13.7 (95\% CI: [12.3, 15.2]), respectively ( $\mathrm{p}=0.403)$. The mean scores for "temporal demand" were 7.8 (95\% CI: $[6.1,10.1])$ with tactile feedback and 7.8 (95\% CI: $[6.0,10.0])$ without $(p=0.564)$. The mean scores for "performance" were $14.6(95 \% \mathrm{CI}:[13.2,16.2])$ and 14.6 (95\% CI: $[13.3,16.1])$, respectively, with versus without tactile feedback $(\mathrm{p}=1.000)$. The mean scores for "effort" were 11.1 (95\% CI: [9.5, 12.9]) with and 11.8 (95\% CI: [10.3, 13.5]) without tactile feedback $(\mathrm{p}=0.025)$. The mean scores for "frustration" were $5.6(95 \% \mathrm{CI}:[4.1,7.6])$ and $5.4(95 \% \mathrm{CI}$ : $[4.3,6.8])$, respectively, with versus without tactile feedback $(\mathrm{p}=0.780)$.

The confidence intervals and means values of the NASA TLX categories for the touch-typing trials are summarized in Figure 24. The mean scores for "mental demand" were 9.2 (95\% CI: [6.9, 12.2]) with tactile feedback and $9.8(95 \% \mathrm{CI}:[7.4,12.9])$ without $(\mathrm{p}=0.001)$. The mean scores for "physical demand" were 13.1 (95\% CI: [11.3, 15.1]) and 13.0 (95\% CI: [11.2, 15.1]), respectively, with and without tactile feedback $(\mathrm{p}=0.561)$. The mean scores for "temporal demand" were 8.5 (95\% CI: $[6.6,10.9])$ with and $8.7(95 \%$ CI: $[6.8,11.2])$ without tactile feedback $(\mathrm{p}=0.176)$. The mean scores for "performance" were 12.3 (95\% CI: [10.4, 14.7]) with tactile feedback and 11.8 (95\% CI: [10.0, 14.0]) without $(\mathrm{p}=0.125)$. The mean scores for "effort" were 13.1 (95\% CI: $[11.4,15.0])$ with and 14.6 (95\% CI: [13.3, 16.0]) without tactile feedback $(\mathrm{p}<0.001)$. The mean scores for "frustration" were 9.2 (95\% CI: [7.0, $12.1])$ and 10.7 (95\% CI: [8.5, 13.4]), respectively, with and without tactile feedback $(p=0.015)$. 


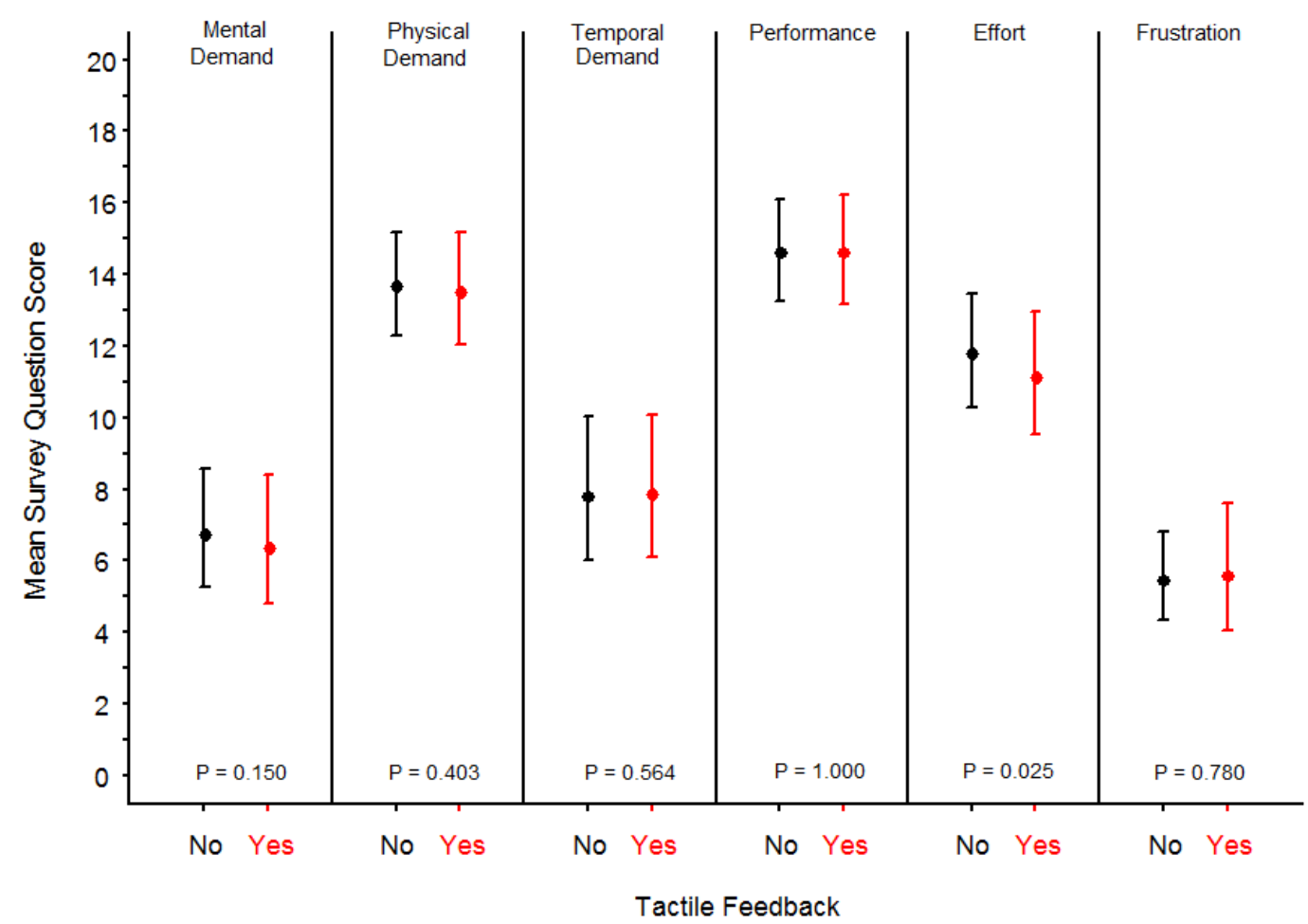

Figure 23.-Hunt-and-peck TLX summary.

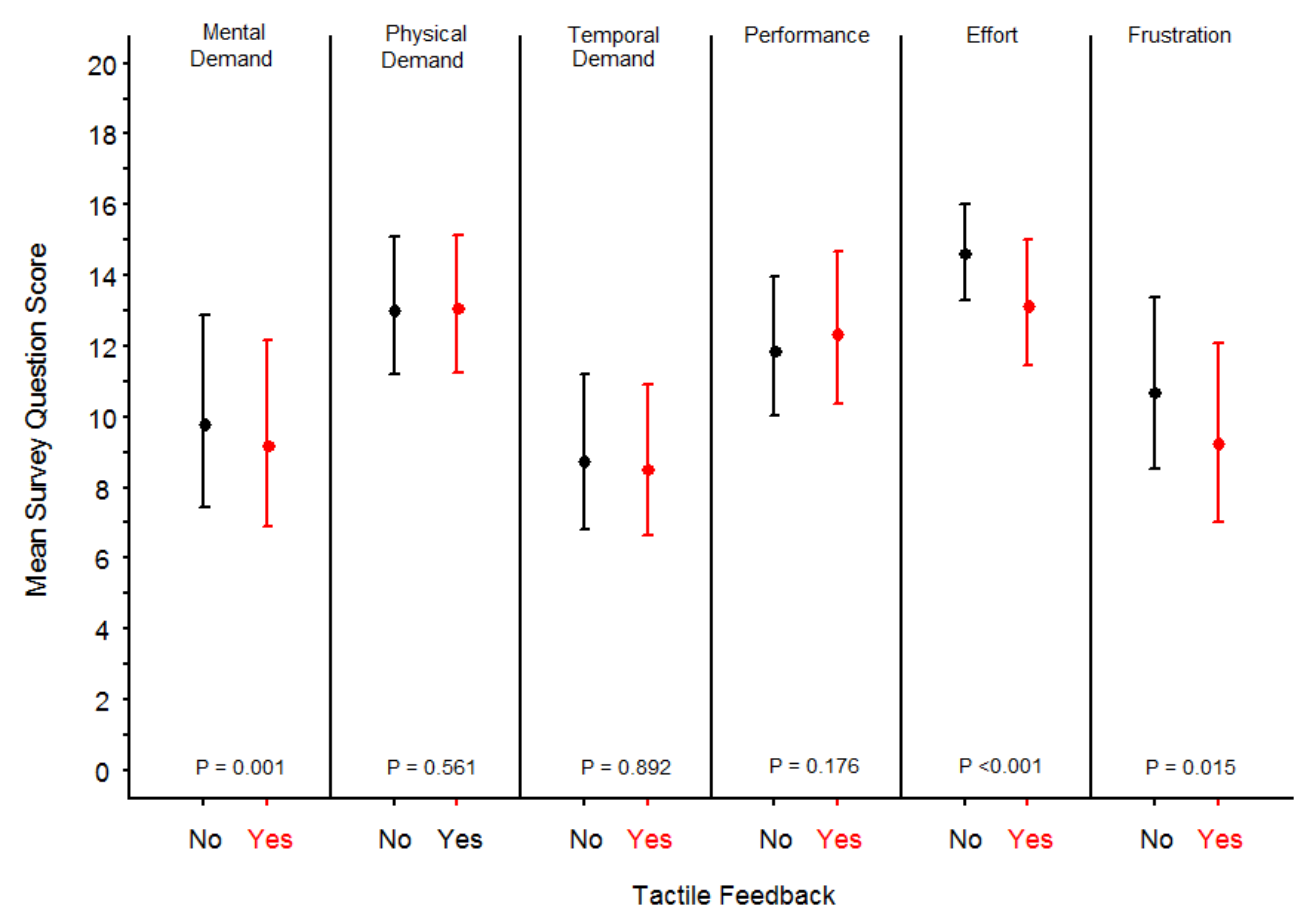

Figure 24.-Touch-typing TLX summary. 


\subsection{Discussion}

Despite the stiffness and bulk of the pressurized gloves, users were able to employ the GECO system to accurately generate desired finger strike sequences, both with and without tactile feedback. In both cases the lower bound on a 95\% CI (over all subjects and trials) exceeds 95\% accuracy. Subjects, with only limited practice, were remarkably effective. Individually, none of the 20 participants achieved a predicted mean below $88 \%$ accuracy. Five of the subjects achieved a predicted mean accuracy of greater than $99 \%$ for both tactile and non-tactile conditions. It may be reasonably argued that highly skilled astronauts might fall into this highest-performing group.

Over the ensemble of subjects, and for every one of the 20 individual participants, mean performance improved with tactile feedback; however the effect cannot be considered statistically significant. The unexpectedly high level of performance across both conditions likely resulted in a ceiling effect that reduced the power of the test to detect differences due to tactile condition.

Previous research has found that due to natural hand synergies, normal subjects attempting to move a single finger will produce motion in others (Ref. 18). Expected error rates were therefore expected to vary as a function of the finger targeted. Additionally, the bulk of the surrogate TMG layer causes some degree of binding between fingers that could contribute to a finger-dependency in error rate. Indeed, $61.9 \%$ (95\% CI: $[55.6,68.0 \%])$ of errors involved an erroneous strike by a finger adjacent to the targeted digit. Figure 10 shows that there was a significantly greater chance of errors targeting either the ring or little finger versus the thumb, index, or middle. Nevertheless, the predicted mean accuracy in these cases was 94.1\% (95\% CI: [92.0, 95.7\%]) for the tactile case, and 92.9\% (95\% CI: [90.7, 94.6\%]) (p<0.001) in the non-tactile condition. Considering that some subjects achieved even higher accuracy rates, the ring and little fingers can be considered useful for the GECO human-computer interface.

In investigating the impact of tactile feedback on participants' accuracy in generating finger strikes, only the first three sequences in any game were included in the primary analysis. This decision was informed in part by a previous study (Ref. 19), in which 24 elderly hearing-impaired and 12 young normal-hearing individuals performed a Simon game-like activity. Both groups' success rate approached $100 \%$ for sequences of length three or less, after which an accuracy drop-off occurred related to working memory and sequence learning ability. To explore this effect, secondary analyses in the GECO study included the impact of sequence length. Figure 12 shows that for both the tactile and non-tactile conditions, the mean accuracy of finger strikes for target sequences of 3 or fewer was greater than for target sequences of 4 or 5 . In both cases the effect size was small, approximately $2 \%$, but significant, confirming the original conjecture on interference due to memory/learning effects beyond the third sequence. A future study is under consideration to specifically explore the potential benefit of tactile feedback for memory/learning.

Shoulder and arm fatigue was noted by some participants in the Phase I study (Ref. 11), motivating inquiry into potential degradation in user performance over the course of the 20 Simon games played. The same Phase I study detected a significant training effect that resulted in increased performance in a textual data entry task over time. Regression analysis of the Simon Game data revealed no significant relationship between game number and accuracy, indicating either (1) both the absence of a significant training effect (beyond the 8 practice games) as well as absence of a significant fatigue effect on performance over the games recorded, or (2) that the two effects were present but cancelled each other out. The lack of any observable trend in the summary data in Figure 13 would imply the former. It is likely that onset of significant fatigue would appear some number of games beyond the 28 total played in the GECO protocol. A future study may be conducted to assess how long users could use the gloves prior to fatigue onset.

The mean responses to the subjective Simon Game Likert-scale NASA TLX questionnaire all trended favorably for the tactile case. Taking the six questionnaire response items individually, no claim of significance can be made. Categorizing responses into objective task demand-related and subjective behavioral-related items (Ref. 17) permits a composite view of potential impacts on workload. The composite sum of the three objective task demand items does not show a significant effect due to tactile 
condition. In contrast, the composite of the two negative subjective behavioral items (effort and frustration) reveals a significant benefit for tactile feedback. This finding might indicate that affect (feelings or emotional responses) rather than effect (objective performance) is the most important contribution of tactile feedback in this application.

Written narrative comments included statements that tactile feedback provided a more "natural," "intuitive," and or "pleasurable" interface; and made the task "easier." A number of participants made remarks similar to these samples: "no feedback made me want to push harder to ensure registration," "it was much easier to know when a button was pushed with the tactile feedback," and "vibration helped verify contact." One respondent took a contrary view, "was almost oblivious to the tactile feedback didn't notice it there or not." Although numerous participants noted that the gloves were "too stiff" (an unavoidable consequence of pressurization), there were surprisingly no complaints about hand fatigue in the Simon Game.

The estimated effect size for tactile feedback ( $8 \%$ improvement) in relation to text entry performance was consistent with previous investigations involving smartphones (Ref. 20) and unpressurized gloves (Ref. 11). In the GECO evaluation, tactile feedback provided a statistically significant benefit for data entry rate (CPM) as well as error rate (KPC) across data entry modes. A reduction in MSD error rate in the touch-type mode with active tactile feedback also suggests that unintended key strokes may go undetected without the cues. In the subjective workload measures assessed in the TLX Likert instrument, tactile feedback showed a benefit for "effort" level in each data entry modality. In touch-typing, tactile feedback also resulted in lower "mental demand" and "frustration." It is interesting to note that while participants performed significantly better with tactile feedback in the objective measures, TLX results indicate they didn't overtly perceive this improved performance. This might be evidence of unconscious somatosensory processing of tactile information (Ref. 21) in the higher mental workload text entry activity.

The hunt-and-peck mode resulted in clearly superior text entry performance compared to the touchtype approach, (35\% greater) and was associated with fewer errors. The similarity of the task to texting on a smart phone may have aided users' rapid adaptation and success. The touch-type mode required familiarity with conventional touch typing hand movements, and was generally less intuitive for the untrained user. Limitations on ease, range, and linearity of motion imposed by the gloves and arm sections within the pressurized glove box appeared to have a greater impact on the touch-type mode which, in this implementation, required greater translational movement than hunt-and-peck.

Multiple participants cited the convenience of having the thumb "hard-wired" to the space bar in touch-type mode, and expressed interest in having the same in hunt-and-peck. This has motivated the creation of a new "hybrid" MOUSE mode which implements this concept. The hybrid mode also hardwires the middle, ring, and little fingers to the backspace key; in recognition of the synergies observed in Figure 10. Based on the Simon Game results, we can expect accuracies of between 96.2 and 98.1\% ( $95 \%$ confidence) for first-time untrained users in this mode. Text completion keys have also been added to the virtual keyboard to accelerate entry rates.

The emphasis on textual data entry in this study is not meant to imply that it is the only, or even primary, use case for the GECO system. Texting capability does directly enable new forms of EVA communications, for example text "chat" communications. In the consumer market, despite the pervasive availability of voice, texting has proven to be an enduring, and arguable pre-eminent, form of interperson tele-discourse. The time-delays imposed by deep-space missions, limited bandwidth, and cluttered audio channels make texting perhaps even more relevant for EVA. Text entry is also relevant for scientific note taking, cataloguing, and annotation of samples/images. Beyond text, GECO's ability to emulate the ubiquitous mouse, in the most literal sense, makes its utility equally as broad. Mouse emulation enables manipulation of buttons, sliders, drop-down menus, toolbars, and/or any other conceivable GUI widget. In the most general sense, the ten fingers of the GECO gloves represent ten buttons that can be mapped to any function. The GECO system could thus replace cumbersome on-suit elements, many of which date to the Apollo-era, as the primary controls for environmental systems (retaining the physical controls as backups). Likewise, GECO could replace difficult-to-access keypads or 
switches on EVA crew exploration systems. Astronauts donning suits through the suit ports in the Space Exploration Vehicle (SEV) could immediately gain access, through the GECO gloves, to any required information and control systems.

On-going efforts include pressurized testing of right- and left-hand GECO gloves at NASA Johnson Space Center (JSC) on the Mark III Advanced EVA Space Suit Technology Demonstrator, scheduled for late-March 2013. Suited testing will support improved assessment of arm mobility impacts on users' ability to employ the GECO gloves for data entry. Evaluations at JSC will also include use of EVA tools in canonical tasks to characterize the performance of GECO gloves for manual manipulation activities. Future plans may include operational and analog environment testing in "Rockyard" and/or Desert Research and Technology Studies evaluations (Ref. 22).

Eventual incorporation of GECO technology into a future suit is subject to future decisions on the direction of EVA technology. The team will continue to investigate improvements to software, hardware, data entry modalities, and suit integration concepts. The final configuration of an operational GECO system will be determined by broader decisions involving elements such as suit-integrated graphical display, EVA computing and communications architectures, as well as operational concepts of employment. These may drive alternative realizations of GECO capabilities such as hand motion tracking. IR LED tracking may, for example, be replaced by a visible camera-based vision system, suitintegrated joint position sensing, magnetic marker tracking, structured light sensing, laser direction and range finding, and/or intertial sensing. The fundamental concept of employing hand motion tracking, finger movement sensing, and tactile feedback persists in these alternate realizations.

\subsection{Conclusions}

Simon Game and Text Entry experiments of GECO system employment in a glove box at 4.3 psid demonstrate that users can effectively use an instrumented glove as a human-computer interface under an EVA-like pressure differentials. With very little training, volunteers were able to employ a GECO glove, achieving high accuracy in generation of volitional finger strikes. Participants were also able to use the glove to enter text through a virtual keyboard in modes emulating both a conventional mouse and a QWERTY keyboard. Investigation of the impact of vibrotactile feedback showed an improvement for both text entry rate and reduction in errors. Subjective responses to a NASA TLX Likert-scale instrument also revealed a perceived reduction in workload with tactile feedback. The results strongly support the viability of GECO as a potential solution to the yet-unsolved challenge of providing access to suitintegrated computing, communications, and control systems during EVA. Follow-on suited testing will investigate the benefits and challenges of this new technology for human-computer interface.

\section{References}

1. Boucher, M.F., Hodgson, E., Murray, S.K., Lee, P., and Braham, S., "Investigation of EVA Information Interface Technology in a Mars Analog Arctic Field Science Setting," 32nd International Conference on Environmental Systems (ICES), Vancouver, B.C., 2002.

2. Hodgson et al., "Requirements and Potential for Enhanced EVA Information Interfaces," 33rd International Conference on Environmental Systems (ICES), Vancouver, B.C., 2003.

3. Hurlbert, K., Bagdigian, B., Carroll, C., Jeevarajan, A., Kliss, M., and Singh, B., Space Technology Roadmap, Technology Area 06, Human Health, Life Support and Habitation Systems, NASA, Washington, D.C., April 2012.

4. Graziosi, D., Stein, J., Ross, A., and Kosmo, J., "Phase VI Advanced EVA Glove Development and Certification for the International Space Station," 31st International Conference on Environmental Systems (ICES), Tokyo, Japan 2001.

5. Thompson, S., Holden, K., England, S., and Benson, E., "The Effect of Pressurized Space Gloves on Operability of Cursor Controls, Mobility, and Strength," Proceedings of the Human Factors and Ergonomics Society 55th Annual Meeting, Las Vegas, NV, 2011. 
6. Wickens, C.D., "Multiple Resources and Mental Workload," Human Factors, vol. 50, no. 3, June 2008.

7. Stolen, M.F., Dillow, B., Jacobs, S.E., Akin, D.L., "Interface for EVA Human-Machine Interaction," 38th International Conference on Environmental Systems, SAE Paper 2008-01-1986.

8. Jacobs, S.E., Di Capua, M., Husain, S.A., Mirvis, A., Akin, D.L., "Incorporating Advanced Controls, Displays and Other Smart Elements into Space Suit Design," 39th International Conference on Environmental Systems, SAE Paper 2009-01-2472.

9. Hieronymus, J.L., "Clarissa Spoken Dialogue System for Procedure Reading and Navigation," IAC-04-T.3.07, NASA Ames Research Center, USA, 2004.

10. Rayner, M., Hockey, B.A., Renders, J.M., Chatzichrisafi, N., and Farrel, K., "Spoken Language Processing in the Clarissa Procedure Browser," Natural Language Engineering, 1, Cambridge University Press, U.K., 2005.

11. Adams, R.J., Olowin, A.B., Hannaford, B., and Sands, O., "Tactile Data Entry for Extravehicular Activity," 2011 IEEE World Haptics Conference (WHC), Istanbul, TK, June 2011.

12. Lindsay, J., Adams, R., and Hannaford, B., "Improving Tactile Feedback With an Impedance Adapter," 2013 IEEE World Haptics Conference (WHC), Daejeon, Korea, April 2013.

13. Soukoreff, R.W. and MacKenzie, I.S., "Metrics for Text Entry Research: An Evaluation of MSD and KSPC, and a New Unified Error Metric," CHI 2003, Ft. Lauderdale, FL, 2003.

14. Humes, L.E. and Floyd, S.S., "Measures of Working Memory, Sequence Learning, and Speech Recognition in the Elderly," Journal of Speech, Language, and Hearing Research, vol. 48, pp. 224235, Feb 2005.

15. Hardin, J. and Hilbe, J., Generalized Estimating Equations, Chapman and Hall/CRC, London, 2003.

16. Fitzmaurice, G., Laird, N., and Ware, J., Applied Longitudinal Analysis, Wiley-Interscience, Hoboken, NJ, 2004.

17. Hart, S.G. and Staveland, L.E. "Development of NASA-TLX: Results of Empirical and Theoretical Research," In P.A. Hancock \& N. Meshkati (Eds.), Human Mental Workload, Amsterdam: NorthHolland, 1988, pp. 139-183.

18. Hager-Ross, C. and Schieber, M.H., "Quantifying the Independence of Human Finger Movements: Comparisons of Digits, Hands, and Movement Frequencies," Journal of Neuroscience, vol. 20, no. 22, pp. 8542-8550, 2000.

19. Humes, L.E. and Floyd, S.S., "Measures of Working Memory, Sequence Learning, and Speech Recognition in the Elderly," Journal of Speech, Language, and Hearing Research, vol. 48, pp. 224235, Feb 2005.

20. Brewster, S., Chohan, F., and Brown L., "Tactile Feedback for Mobile Interactions," Proceedings of the SIGCHI Conference on Human Factors in Computing Systems, 2007.

21. Dijkerman, C.H. and de Haan, E.H.F., "Somatosensory Processes Subserving Perception and Action," Behavioral and Brain Sciences, vol. 30, 189-239, 2007.

22. Ross, A., Kosmo, J., and Janoiko, B., "Historical Synopses of Desert RATS 1997-2010 and a Preview of Desert RATS 2011," Acta Astronautica, May 2012. 

